\title{
Reciprocal Disruptions in Thalamic and Hippocampal Resting-State Functional Connectivity in Youth with 22q11.2 Deletions
}

\author{
Charles Schleifer ${ }^{1}$, Amy Lin ${ }^{2.3}$, Leila Kushan ${ }^{2}$, Jie Lisa $\mathrm{Ji}^{1,4}$, Genevieve Yang ${ }^{1,5}$, Carrie E. Bearden*2,3,4 ${ }^{2}$ Alan \\ Anticevic $*, 1,5,6,7,8$
}

1. Department of Psychiatry, Yale University School of Medicine, 300 George Street, New Haven, CT 06511, USA

2. Department of Psychiatry and Biobehavioral Sciences, Semel Institute for Neuroscience and Human Behavior, University of California at Los Angeles, Los Angeles, CA 90095, USA

3. Interdepartmental Neuroscience Program, University of California at Los Angeles, Los Angeles, CA 90095, USA

4. Department of Psychology, University of California at Los Angeles, Los Angeles, CA 90095, USA

5. Interdepartmental Neuroscience Program, Yale University, New Haven, CT 06520, USA

6. Abraham Ribicoff Research Facilities, Connecticut Mental Health Center, New Haven, CT 06519, USA

7. NIAAA Center for the Translational Neuroscience of Alcoholism, New Haven, CT 06519, USA

8. Department of Psychology, Yale University, 2 Hillhouse Avenue, CT 06520, USA

\section{*Co-corresponding}

\section{Corresponding Authors:}

\author{
Alan Anticevic Ph.D., Yale University, Department of Psychiatry \\ 34 Park St., New Haven, CT 06519, Office (203) 974-7763 \\ alan.anticevic@yale.edu \\ Carrie E. Bearden, Ph.D., University of California, Los Angeles \\ Semel Institute for Neuroscience and Human Behavior \\ A7-460 Semel Institute, 760 Westwood Plaza, LA, CA 90095 \\ cbearden@mednet.ucla.edu
}

Author Contributions: (using CRediT Taxonomy, http://www.cell.com/pb/assets/raw/shared/guidelines/CRediT-taxonomy.pdf): Conceptualization, C.E.B., A.A. Methodology, C.E.B., J.L.J., A.L. C.S., A.A.; Formal Analysis, C.S., A.A., J.L.J., G.Y..; Data Curation, C.S., A.L., A.A., C.E.B..; Visualization, C.S., A.A., J.L.J..; Writing - Original Draft, A.A., C.S., A.L., C.E.B. Writing Review \& Editing, C.S., A.A., C.E.B.; Supervision, A.A., C.E.B.

Acknowledgements: Aspects of the data were provided by the Human Connectome Project, WU-Minn Consortium (Principal Investigators: David Van Essen and Kamil Ugurbil; 1U54MH091657) funded by the 16 NIH Institutes and Centers that support the NIH Blueprint for Neuroscience Research; and by the McDonnell Center for Systems Neuroscience at Washington University. This work was supported by the NIH via awards DP5-OD012109 (Anticevic), R01-MH108590 (Anticevic), R01-MH112189 (Anticevic), R01 MH085953 (Bearden), U54 EB020403 (Bearden), T32MH073526 (Lin), as well as the Brain and Behavior Foundation (NARSAD) Independent Investigator grant (Anticevic) and the Joanne and George Miller Family Endowed Term Chair (Bearden).

Conflict of Interest: A.A. consults and is a SAB member for BlackThorn Therapeutics Inc. Other authors have no conflicts to report. 


\begin{abstract}
22q11.2 deletion syndrome (22q11DS) is a recurrent copy number variant (CNV) with high penetrance for developmental neuropsychiatric disorders. Study of individuals with 22q11DS therefore may offer key insights into neural mechanisms underlying such complex illnesses. Resting-state functional MRI (rs-fMRI) studies in idiopathic schizophrenia have consistently revealed disruption of thalamic and hippocampal circuitry. Here, we sought to test whether this circuitry is similarly disrupted in the context of this genetic high-risk condition. To this end, resting-state functional connectivity patterns were assessed in a sample of young men and women with 22q11DS $(n=42)$ and demographically matched healthy controls $(n=39)$. Neuroimaging data were acquired via single-band protocols, and analyzed in line with methods provided by the Human Connectome Project (HCP). We computed functional relationships between individual-specific anatomically-defined thalamic and hippocampal seeds and all gray matter voxels in the brain. Whole-brain type I error protection was achieved through nonparametric permutation-based methods. 22q11DS patients displayed reciprocal disruptions in thalamic and hippocampal functional connectivity relative to control subjects. Thalamo-cortical coupling was increased in sensorimotor cortex, and reduced across associative networks. The opposite effect was observed for the hippocampus in regards to sensory and associative network connectivity. The thalamic and hippocampal dysconnectivity observed in 22q11DS suggest that high genetic risk for psychiatric illness is linked with disruptions in large-scale cortico-subcortical networks underlying higher-order cognitive functions. These effects highlight the translational importance of large-effect CNVs for informing mechanisms underlying neural disruptions observed in idiopathic developmental neuropsychiatric disorders.
\end{abstract}

\title{
SIGNIFICANCE STATEMENT
}

Investigation of neuroimaging biomarkers in highly penetrant genetic syndromes represents a more biologically tractable approach to identify neural circuit disruptions underlying developmental neuropsychiatric conditions. 22q11.2 deletion syndrome confers particularly high risk for psychotic disorders, and is thus an important 
translational model in which to investigate systems-level mechanisms implicated in idiopathic illness. Here, we show resting-state fMRI evidence of large-scale sensory and executive network disruptions in youth with 22q11DS. In particular, this study provides the first evidence that these networks are disrupted in a reciprocal fashion with regard to the functional connectivity of the thalamus and hippocampus, suggesting circuit-level dysfunction.

\section{INTRODUCTION}

Remarkable genetic and clinical heterogeneity presents a challenge for mapping pathological processes underlying neuropsychiatric disorders such as schizophrenia and autism spectrum disorder (ASD). These disorders are increasingly viewed as developmental disruptions of neural circuitry with major genetic contributions (Insel, 2010; Geschwind and Flint, 2015). Thus, genetically-defined syndromes with strong predisposition for neuropsychiatric illness provide powerful models to elucidate neural mechanisms underlying these complex disorders.

22q11.2 Deletion Syndrome (22q11DS), also known as DiGeorge or Velocardiofacial syndrome (OMIM \#188400, \#192430), occurs in about 1 in 4,000 live births (McDonald-McGinn et al., 2015). It represents one of the greatest known genetic risk factors for psychosis, approximately 25 times population base rates (Bassett and Chow, 2008; Green et al., 2017), while additionally conferring elevated risk for multiple childhood disorders including attention deficit hyperactivity disorder, anxiety disorder, and ASD (Schneider et al., 2014).

Genes within the 22q11.2 locus are implicated in cortical circuit formation and functioning (Meechan et al., 2015; Paronett et al., 2015). Disrupted cortical interneuron migration has been observed in a 22q11.2 mouse model (Meechan et al., 2012; Toritsuka et al., 2013). Correspondingly, deletion carriers present with a range of structural and functional brain abnormalities, including cortical surface area reductions, altered white-matter microstructure (Kates et al., 2001; Jalbrzikowski et al., 2014; Schmitt et al., 2015), and, importantly, disruptions of large-scale network connectivity (Debbane et al., 2012; Padula et al., 2015). Recently, an independent 
components analysis approach revealed significant hypo-connectivity relative to controls within the anterior cingulate/precuneus and default mode networks, which reliably predicted 22q11DS case-control status in an independent cohort (Schreiner et al., 2017). Critically, due to its well-characterized genetic etiology, circuitlevel abnormalities associated with 22q11.2 deletions can be experimentally manipulated in animals to generate causal links with circuit dysfunction. In humans, 22q11DS presents a compelling genetic high-risk model in which anomalous circuitry can be investigated prior to development of overt illness.

Specifically, aberrant connectivity of two key anatomically inter-connected structures, the thalamus and hippocampus, has been implicated in neuropsychiatric disorders (Brown et al., 2017) and schizophrenia in particular (Samudra et al., 2015). The thalamus serves as a critical hub for flow of sensory and higher-order information, facilitating information integration across cortical networks (Guo et al., 2017; Hwang et al., 2017). Consistent alterations of thalamo-cortical circuitry, involving a pattern of prefrontal-thalamic hypo-connectivity, concomitant with somatomotor-thalamic hyper-connectivity, have been identified in schizophrenia patients and at-risk youth (Welsh et al., 2010; Woodward et al., 2012; Anticevic et al., 2014). Similarly, the hippocampus features prominently in schizophrenia neurobiology (Weinberger, 1987). Post-mortem schizophrenia studies have demonstrated hippocampal alterations in excitatory pyramidal cells and local inhibitory interneurons. Hippocampal-prefrontal dysconnectivity during cognitive processing has been proposed as a translational phenotype for schizophrenia, as evidenced by a 22q11 mouse model (Mukai et al., 2015) and by findings of altered connectivity in those at familial high risk for the illness (Meyer-Lindenberg, 2010). Critically, the thalamus and hippocampus exhibit opposing resting-state connectivity patterns in healthy adults (Stein et al., 2000), which would predict distinct alterations in a genetic risk model based on a CNV that disrupts neural circuits. Yet, these translational neural phenotypes have not been investigated in a genetic risk model such as 22q11DS.

Here we take a hypothesis-based approach to study large-scale network alterations in 22q11DS by leveraging findings from animal models of the disorder and imaging work in humans. Using the Human 
Connectome Project analytical pipeline, which yields exceptional cortical spatial alignment (Glasser et al., 2013), we computed functional relationships between subject-specific anatomically-defined thalamic and hippocampal seeds in 22q11DS youth and matched controls. Relative to controls, 22q11DS youth exhibited thalamo-cortical hyper-connectivity with sensorimotor cortex but hypo-connectivity with associative networks. An opposing (i.e. interactive) pattern was found for hippocampal-cortical circuitry, supporting a 22q11DS neural phenotype with distinct effects on thalamic and hippocampal circuits.

\section{METHODS}

Participants. The total sample consisted of 81 participants ( 7 to 26 years of age; 42 22q11DS and 39 demographically matched healthy controls), recruited from an ongoing longitudinal study at the University of California, Los Angeles (UCLA). 22q11DS participants all had a molecularly confirmed 22q11.2 deletion (see Table 1 for demographic details). Exclusion criteria for all study participants were: neurological or medical condition disorder that might affect performance, insufficient fluency in English, and/or substance or alcohol abuse and/or dependence within the past 6 months. Healthy controls (HCS) additionally could not meet diagnostic criteria for any major mental disorder, based on information gathered during administration of the Structured Clinical Interview for DSM-IV Axis I Disorders (First et al., 1996). After study procedures had been fully explained, adult participants provided written consent, while participants under the age of 18 years provided written assent with the written consent of their parent or guardian. The UCLA Institutional Review Board (IRB) approved all study procedures and informed consent documents. 


\begin{tabular}{|c|c|c|c|c|c|c|c|}
\hline & \multicolumn{2}{|c|}{$\operatorname{HCS}(\mathrm{N}=39)$} & \multicolumn{3}{|c|}{$22 q 11 D S(N=42)$} & \multirow[b]{2}{*}{$\mathrm{p}$ value } & \\
\hline & M & S.D. & M & S.D & $\mathrm{T}$ value & & \\
\hline Age (yrs) & 14.1 & 4.7 & 15.7 & 5.3 & 1.5 & 0.140 & \\
\hline Sex $(\%$ male $)$ & 46.2 & 0.5 & 40.5 & 49.7 & 0.5 & 0.612 & \\
\hline Paternal Education (yrs) & 5.9 & 1.9 & 6.5 & 1.8 & 1.6 & 0.111 & \\
\hline Maternal Education (yrs) & 6.3 & 1.8 & 6.8 & 1.1 & 1.6 & 0.122 & \\
\hline Subject Education (yrs) & 7.9 & 4.7 & 8.5 & 3.8 & 0.7 & 0.499 & \\
\hline Handedness (\% right handed) & 90.5 & 0.3 & 95.2 & 21.6 & 0.8 & 0.416 & \\
\hline WASI Full Scale IQ & 108.0 & 20.2 & 77.6 & 14.5 & 7.3 & $1.86 \times 10^{-10}$ & $* * *$ \\
\hline Verbal IQ & 57.8 & 13.9 & 36.2 & 9.6 & 7.6 & $5.55 \times 10^{-11}$ & $* * *$ \\
\hline Nonverbal IQ & 50.0 & 11.4 & 34.4 & 13.4 & 5.6 & $3.31 \times 10^{-7}$ & $* * *$ \\
\hline $\begin{array}{l}\text { Antipsychotic ( } \% \text { of subjects } \\
\text { medicated) }\end{array}$ & 0 & -- & 12 & -- & -- & -- & \\
\hline $\begin{array}{l}\text { Psychostimulant (\% of subjects } \\
\text { medicated) }\end{array}$ & 0 & -- & 14 & -- & -- & -- & \\
\hline $\begin{array}{l}\text { Prodromal Syndrome }(\% \text { of } \\
\text { subjects meeting COPS) }\end{array}$ & 0 & -- & 35.7 & -- & -- & -- & \\
\hline $\begin{array}{l}\text { BOLD Movement ( } \% \text { frames } \\
\text { scrubbed) }\end{array}$ & 5.0 & 9.8 & 4.4 & 6.8 & 0.3 & 0.755 & \\
\hline BOLD Signal-to-Noise Ratio & 89.0 & 14.6 & 92.1 & 15.4 & 0.9 & 0.369 & \\
\hline
\end{tabular}

Table 1. Demographic and symptom measures for 22q11DS $(n=42)$ and healthy control subject $(n=39)$ groups. IQ: intelligence quotient; WASI: Weschler Adult Intelligence Scale; Verbal $\mathrm{IQ}=$ WASI Vocabulary T-score; Nonverbal IQ= WASI Matrix Reasoning T-score; COPS: Criteria of Prodromal Syndromes, as part of the Structured Interview for Prodromal Syndromes (SIPS).

Neuroimaging Acquisition. All subjects were imaged on a 3-Tesla Siemens TimTrio scanner with a 32channel phased array head coil at the UCLA Center for Cognitive Neuroscience (CCN). Resting BOLD images were acquired in 34 interleaved axial slices parallel to the anterior-posterior commissure (AC-PC) using a fast gradient-echo, echo-planar sequence $[$ voxel size $=3 \times 3 \times 4 \mathrm{~mm}$, time repetition $(\mathrm{TR})=2000 \mathrm{~ms}$, time echo $(\mathrm{TE})=$ $30 \mathrm{~ms}$, flip angle $=90^{\circ}$, matrix $=64 \times 64$, field of view $\left.=192 \times 192 \mathrm{~mm}\right]$. Acquisition lasted 5.1 minutes and produced 152 volumes. High-resolution T1w images were collected in 160 sagittal slices via a magnetizationprepared rapid gradient-echo sequence $(\mathrm{MP}-\mathrm{RAGE})[$ voxel size $=1 \times 1 \times 1 \mathrm{~mm}, \mathrm{TR}=2300 \mathrm{~ms}, \mathrm{TE}=2.91 \mathrm{~ms}$, flip angle $=9^{\circ}$, matrix $=240 \times 256$, field of view $\left.=240 \times 256 \mathrm{~mm}\right]$ 
Clinical Assessment. On the same day as the scan, demographic information and clinical measures were collected for each participant by trained master's level clinicians (see Table 1). Verbal IQ was assessed via the Wechsler Abbreviated Scale of Intelligence (WASI) Vocabulary subtest and Non-verbal IQ was assessed via the WASI Matrix Reasoning subtest. Psychiatric and dimensional psychotic-like symptoms were assessed via the Structured Interview for Prodromal Symptoms (SIPS; Tandy J. Miller et al., 2002). See (Jalbrzikowski et al., 2012; Jalbrzikowski et al., 2013) for more details on study ascertainment and recruitment procedures.

Data Preprocessing. Structural and functional MRI data were first preprocessed according the methods provided by the Human Connectome Project (HCP), outlined below, and described in detail by the WU-Minn HCP consortium (Glasser et al., 2013). These open-source HCP algorithms, which we further optimized for compatibility with legacy single-band data in this study, represent the current state-of-the-art approaches in spatial distortion correction, registration, and maximization of high-resolution signal-to-noise (SNR) (Glasser et al., 2016). All processing methods closely followed the minimal processing pipelines as outlined by Glasser and colleagues (Glasser et al., 2013), with a few key modifications.

The adapted HCP pipeline included the following steps: i) the T1-weighted images were corrected for bias-field distortions and warped to the standard Montreal Neurological Institute-152 (MNI-152) brain template through a combination of linear and non-linear transformations using the FMRIB Software Library (FSL) linear image registration tool (FLIRT) and non-linear image registration tool (FNIRT) (Jenkinson et al., 2002). ii) FreeSurfer's recon-all pipeline was employed to compute brain-wide segmentation of gray and white matter to produce individual cortical and subcortical anatomical segmentation (Reuter et al., 2012). iii) Next, cortical surface models were generated for pial and white matter boundaries as well as segmentation masks for each subcortical grey matter voxel. Using the pial and white matter surface boundaries, a 'cortical ribbon' was defined along with corresponding subcortical voxels, which were combined to generate the Connectivity Informatics Technology Initiative (CIFTI) volume/surface 'gray-ordinate' space for each individual subject, 
which drastically reduces file management for combined surface and volume analyses and visualization and establishes a combined cortical surface and subcortical volume coordinate system (Glasser et al., 2013). iv) the cortical surfaces were then registered to the group average HCP atlas using surface-based registration based on cortical landmark features, whereas the subcortical 'volume' component of the image was brought into group atlas alignment via non-linear registration (Glasser et al., 2013). v) The BOLD data were motion corrected and aligned to the middle frame of every run via FLIRT. In turn, a liberal brain-mask was applied to exclude signal from non-brain tissue. After initial processing in NIFTI volume space, BOLD data were converted to the CIFTI gray matter matrix by sampling from the anatomically-defined gray matter cortical ribbon whereas the subcortical voxels were isolated using subject-specific FreeSurfer segmentation. The subcortical volume component of the BOLD data was then aligned to the group atlas as part of the NIFTI processing in a single transform step that concatenates all of the transform matrixes for each prior processing step (i.e. motion correction, registration, distortion correction). This produced a single nonlinear transformation to minimize interpolation cost. In turn, the cortical surface component of the CIFTI file was aligned to the HCP atlas using surface-based nonlinear deformation based on sulcal features.

Following these 'minimal' HCP preprocessing steps, a high-pass filter $(>0.5 \mathrm{~Hz})$ was applied to the BOLD time series in order to remove low temporal frequencies and scanner drift. In-house MATLAB tools were then used to compute the signal in the ventricles, deep white matter, and across all gray matter voxels (proxy of global mean signal regression to address spatially pervasive sources of artifacts; (Power et al., 2017)). These time series were modeled as nuisance variables and were regressed out of the gray matter voxels. Subsequent analyses used the residual BOLD time series following these de-noising steps.

Functional Connectivity Analyses. Thalamic and hippocampal seeds were first defined individually for each subject through automatic anatomical segmentation of high-resolution structural images via FreeSurfer software as part of the HCP minimal preprocessing pipelines. These structures were then used as 'seeds', as 
conducted in our prior work (Anticevic et al., 2014). Specifically, Pearson correlations were computed between the mean BOLD signal time series in each seed and the BOLD time series at every other cortical and subcortical vertex in CIFTI gray-ordinate space. These correlation maps were then standardized for statistical analyses via Fisher r-to-Z transformation.

As noted, the thalamus and hippocampus in humans exhibit distinct resting-state connectivity profiles (Stein et al., 2000). In fact, this would predict distinct alterations in a genetic risk model based on a de novo CNV that uniformly affects neural circuits. In turn, combining two 'seed' regions, both of which may be affected, but with opposing predicted directions of alterations, constitutes a more powered neural marker. Put differently, we hypothesized a Group by Seed interaction whereby 22q11DS may exhibit distinct bi-directional alterations across the hippocampal and thalamic systems. To confirm the viability of this logic, we computed an a priori quantitative independent test of differences in thalamic and hippocampal connectivity in a sample of 339 unrelated healthy adults derived from the Human Connectome Project (HCP). This provided the basis for the expected interactive effects between thalamic and hippocampal seeds in the core between-group analysis. In other words, the purpose of the HCP dataset here was to serve as a large normative sample to provide an empirical independent basis for the proposed clinical hypotheses.

Next, to test the Group $x$ Seed interaction effect with the BOLD rs-fcMRI as the dependent measure, we computed a two-way repeated measures ANOVA with a factor of Group (22q11DS vs. HCS) and Seed (thalamus vs. hippocampus). Whole-brain type I error protection was applied via non-parametric permutation testing with FSL's Permutation Analysis of Linear Models (PALM) algorithm (Winkler et al., 2014) with 10,000 permutations. This approach circumvents the distributional assumptions (e.g. normality) that may result in type I error inflation (Eklund et al., 2016). We also independently repeated our seed-based analyses in each of seven a priori functional networks described by Yeo and colleagues to test for network specificity of the hypothesized effects (Buckner et al., 2011; Yeo et al., 2011; Choi et al., 2012). 
To quantify the differential contributions of thalamic and hippocampal sub-regions, a k-means algorithm was used to cluster voxels within each seed based on the correlation distance between their grouplevel rs-fcMRI effects. Multiple cluster solutions were possible, but we elected to focus on a parsimonious twocluster solutions for the thalamus and hippocampus because the higher cluster solutions explained proportionally less of the variance, as demonstrated by the 'Elbow Method' (Thorndike, 1953). Seed-based functional connectivity was subsequently computed for each of the four resultant clusters (two per seed). Each cluster's whole-brain connectivity matrix was then correlated with the whole-brain connectivity matrix previously computed for the whole seed. Within the thalamus and hippocampus, the two resulting Pearson coefficients were compared using Steiger's Z-test to determine which cluster's connectivity profile most resembled that of the whole seed (Steiger, 1980). For the thalamus, the connectivity profiles of the whole seed, as well as the anterior and posterior data-derived clusters, were compared to the functional connectivity of seven a priori anatomical seeds derived from the FSL thalamic atlas.

The utility of the observed rs-fcMRI effects for individual classification accuracy was assessed via a supervised binary classification algorithm. A total $n=1000$ iterations of a Support Vector Machine (SVM) were computed, each randomly splitting the $n=81$ pooled subjects and training on $n=41$, then using split-half crossvalidation with the remaining $n=40$ to build a distribution of receiver operator (ROC) curves. One-dimensional SVMs were trained and tested on a single factor consisting of the linear combination of thalamic and hippocampal connectivity to each of the interaction-derived ROIs ([thalROIa + hippROIb] - [thalROIb + hippROIa]). This was repeated for the network-derived results ([thalSOM + hippFPN] - [thalFPN + hippSOM]).

Several analyses were performed in order to address confounds potentially introduced during data acquisition or processing. For BOLD images, frames with significant head movement were flagged based on algorithms and intensity thresholds recommended for multi-band data (Power et al., 2012). Temporal signal-tonoise ratio (SNR) was calculated for each subject as the ratio of mean BOLD signal to its standard deviation 
over time. Movement (percentage of flagged frames) and SNR correlations with rs-fcMRI effects were examined for both groups. To assess medication as a potential confound, two-sample t-tests were computed between rs-fcMRI effects in medicated versus unmediated 22q11DS patients for the subsets taking antipsychotic medications and dopaminergic stimulants. Global signal regression (GSR) was included as a preprocessing step for the main analyses, but functional connectivity was also re-computed for the data without GSR in order to ensure that the effects were comparable at the whole-brain level, and within the specific ROIs derived from permutation testing.

\section{RESULTS}

\section{2q11DS Is Associated with Distinct Functional Dysconnectivity for Thalamus and Hippocampus. As}

noted, we sought to test if 22 q11DS is characterized by disruptions in thalamic as well as hippocampal restingstate functional connectivity (rs-fcMRI). We hypothesized dissociable effects across thalamic and hippocampal seeds, given their known differences in functional connectivity patterns. To establish this effect, we first conducted a 'control' analysis in the $n=339$ healthy adult subjects collected by the HCP (Figure 1). Results showed that the rs-fcMRI profiles of the thalamus and hippocampus are intrinsically anti-correlated with respect to a broad set of regions overlapping with sensory and executive networks.

Next, we tested whether these rs-fcMRI disruptions exhibit interactive effects for 22q11DS versus HCS. As predicted, there were two sets of regions exhibiting a significant 2x2 Group by Seed interaction: i) sensorymotor regions, marked by hyper-connectivity for the thalamus but hypo-connectivity with the hippocampus; ii) a cerebellar region marked by hypo-connectivity for the thalamus but hyper-connectivity with the hippocampus (Figure 2; see Table 4 for all regions surviving type I error correction). Put differently, the interaction was driven by the 22q11DS group exhibiting significantly increased thalamic connectivity (but decreased hippocampal connectivity) with bilateral sensorimotor regions, including the pre- and postcentral gyri and superior temporal gyrus, whereas the opposite effect (decreased thalamic and increased hippocampal 
connectivity) was observed for a region in the left cerebellum. While this effect was localized to the cerebellum following type I error correction, the threshold-free maps show a broader set of prefrontal and parietal regions that trend towards significance (Figure 3).

Ruling out Motion, SNR, and Medication Effects. To ensure that the observed effects were not attributable to differential motion between groups, or to differential SNR profiles, we correlated both measures with the functional connectivity values for both seeds to both interaction-derived ROIs, as well as with the linear combination of these four connectivity values. No significant relationships were observed between functional connectivity and motion or SNR for the 22q11DS or HCS groups (see Table 2). Within the 22q11DS group, mean rs-fcMRI effects were also compared between cohorts of medicated and un-medicated patients (with regards to antipsychotic and stimulant medication). No significant effects of either medication were observed (see Table 3). 
Table 2 - Movement and SNR Relationships

\begin{tabular}{|c|c|c|c|c|c|}
\hline & \multicolumn{2}{|c|}{$\mathrm{HCS}$} & \multicolumn{2}{|c|}{$22 q 11 D S$} \\
\hline & & r value & $\mathrm{p}$ value & r value & $\mathrm{p}$ value \\
\hline \multirow{5}{*}{ 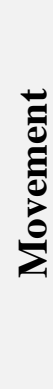 } & Combined rs-fcMRI Effects & 0.09 & 0.60 & 0.26 & 0.09 \\
\hline & Thalamus - ROIa & 0.17 & 0.31 & 0.26 & 0.10 \\
\hline & Thalamus - ROIb & -0.18 & 0.27 & -0.11 & 0.47 \\
\hline & Hippocampus - ROIa & -0.13 & 0.44 & -0.15 & 0.34 \\
\hline & Hippocampus - ROIb & -0.20 & 0.22 & 0.15 & 0.35 \\
\hline \multirow{5}{*}{$\mathbb{Z}_{\mathscr{S}}$} & Combined rs-fcMRI Effects & -0.05 & 0.77 & -0.18 & 0.26 \\
\hline & Thalamus - ROIa & -0.16 & 0.33 & -0.17 & 0.29 \\
\hline & Thalamus - ROIb & 0.02 & 0.89 & -0.05 & 0.75 \\
\hline & Hippocampus - ROIa & 0.01 & 0.95 & 0.09 & 0.59 \\
\hline & Hippocampus - ROIb & 0.04 & 0.81 & -0.23 & 0.15 \\
\hline
\end{tabular}

Table 2. Movement and SNR Relationships. Pearson correlations showing no significant relationship between rs-fcMRI effects (mean Fz connectivity values) and measures of head movement signal-to-noise ratio (SNR). 'Combined fcMRI Effects' refers to the linear combination of connectivity values from the thalamus and hippocampus to ROIa and ROIb ([thalROIa + hippROIb] - [thalROIb + hippROIa]). Connectivity between each seed and ROI (e.g. Thalamus to ROIa) was also individually tested for correlation with motion and SNR. 
Table 3 - Medication Effects. Medicated vs. Un-medicated 22q11DS Patients

\begin{tabular}{lcccc} 
& \multicolumn{2}{c}{ Antipsychotic } & \multicolumn{2}{c}{ Stimulant } \\
\hline & T-Value & p-Value & T-Value & p-Value \\
Combined fcMRI Effects & -1.643 & 0.144 & 0.197 & 0.849 \\
Thalamus - ROIa & -0.342 & 0.735 & -0.581 & 0.581 \\
Thalamus - ROIb & 1.003 & 0.361 & 0.390 & 0.710 \\
Hippocampus - ROIa & -0.560 & 0.603 & -1.002 & 0.346 \\
Hippocampus - ROIb & -1.131 & 0.302 & 0.609 & 0.565 \\
\hline
\end{tabular}

Table 3. Comparison of fcMRI effects in medicated versus un-medicated 22q11DS subjects, with regards to antipsychotics and dopaminergic stimulants. Two-sample t-tests are shown for the linear combination of connectivity values from both seeds and ROIs ([thalROIa + hippROIb] - [thalROIb + hippROIa]), as well as for the connectivity of each individual seed to each ROI. No significant effects of medication were observed. 
a
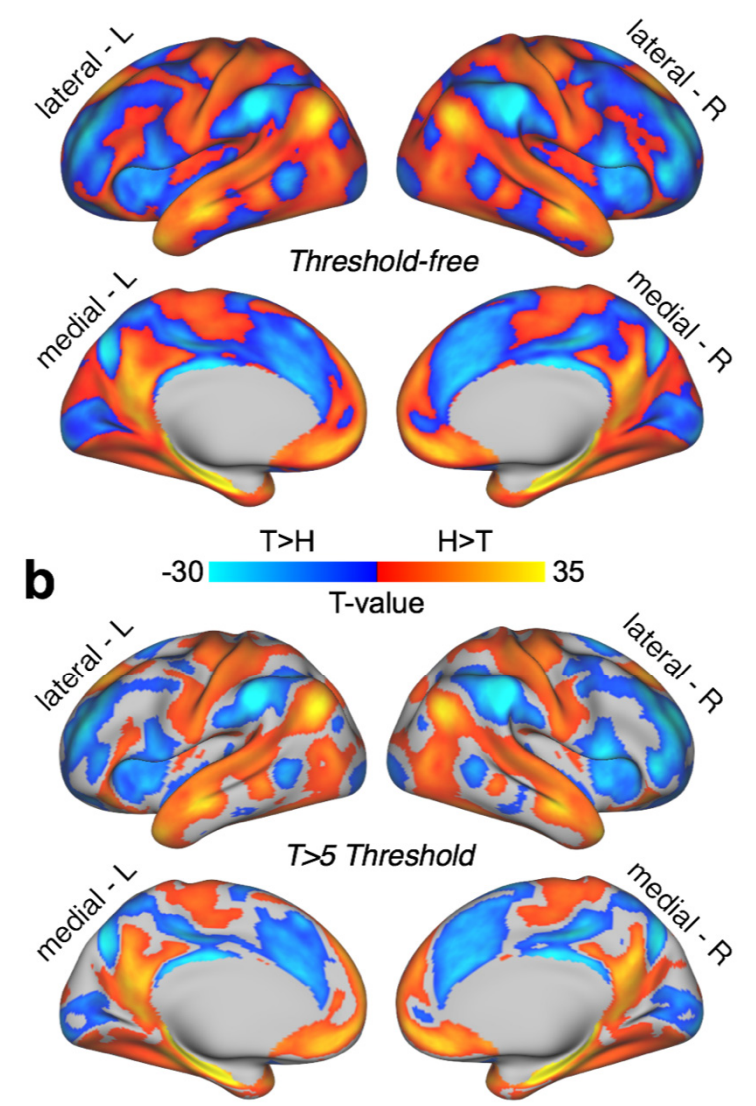

Surface View

$\mathrm{H}>\mathrm{T}$

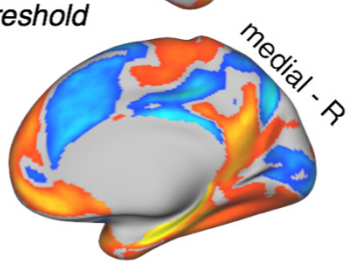

Figure 1. Hippocampal vs. Thalamic Seed Connectivity, N=339 Healthy Adults (HCP Dataset). Comparison of thalamic and hippocampal resting-state functional connectivity in the Human Connectome Project dataset. (a) Surface and volume maps showing the threshold-free dependent-samples t-test between thalamic and hippocampal functional connectivity in $n=339$ healthy adult subjects. (b) The same contrast, masked at T value $>5$. 

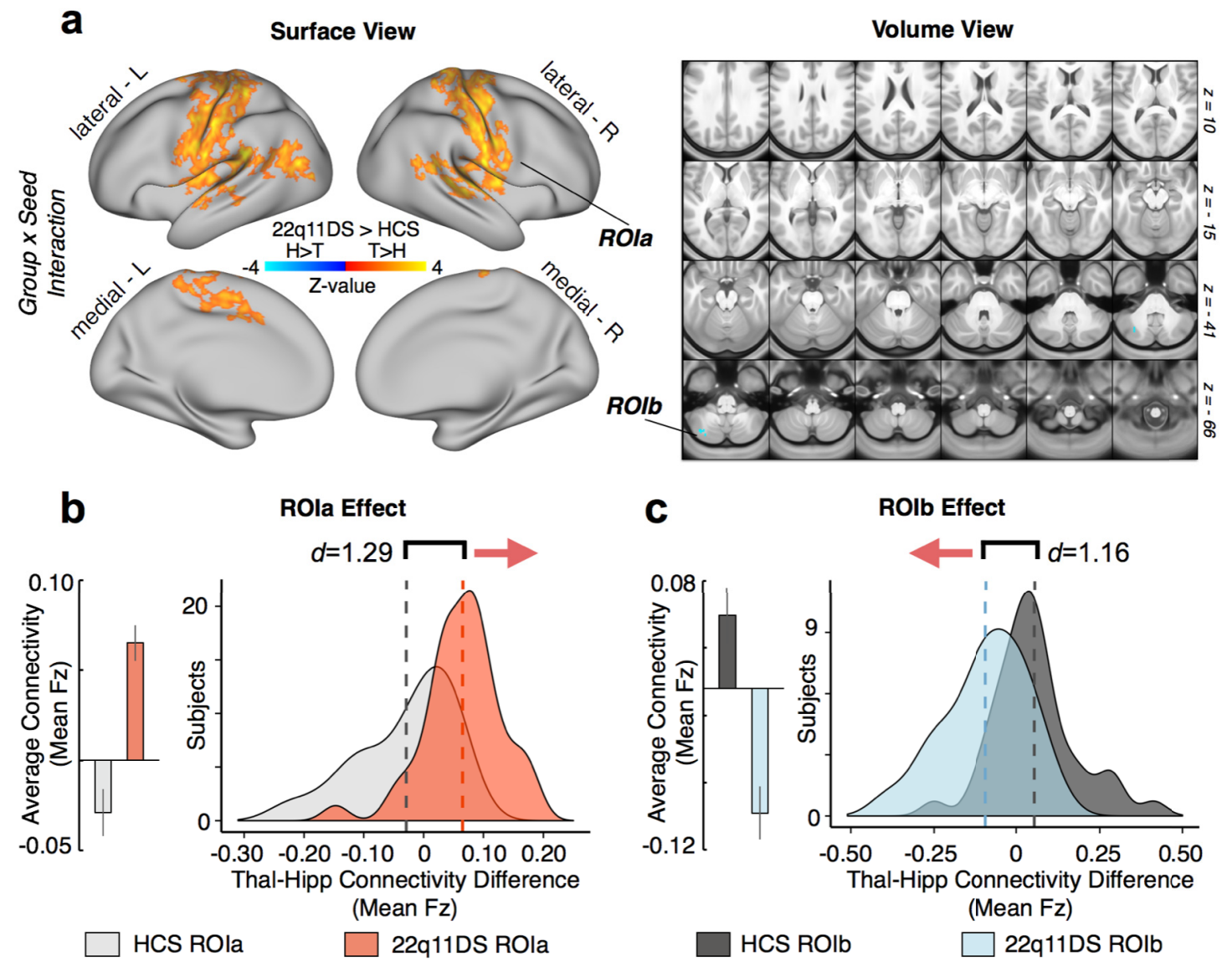

Figure 2. Interaction-Derived Effects. Resting-state functional connectivity of the thalamus and hippocampus in 22q11DS and healthy controls (HCS). (a) Surface and volume maps showing type I error-protected group-level contrast for the 2x2 interaction between Group (22q11DS vs. HCS) and Seed (thalamus vs. hippocampus). Yellow-orange (ROIa) indicates an effect whereby 22q11DS showed thalamic hyper-connectivity but hippocampal hypo-connectivity relative to HCS. The blue contrast (ROIb) indicates an effect whereby 22q11DS showed thalamic hypoconnectivity but hippocampal hyper-connectivity relative to HCS. (b) Difference scores between thalamic and hippocampal connectivity to ROIa (mean Fz) across subjects in each group. Group means (left) and distributions (right) shown to illustrate the direction of the effect. (c) Same as (b) for ROIb, showing an effect in the opposite direction as ROIa. Note: histograms are based on the data extracted from the maps presented in (a). Individual thalamic and hippocampal effects are presented in Figure 10 in comparison with functional network-derived effects. 

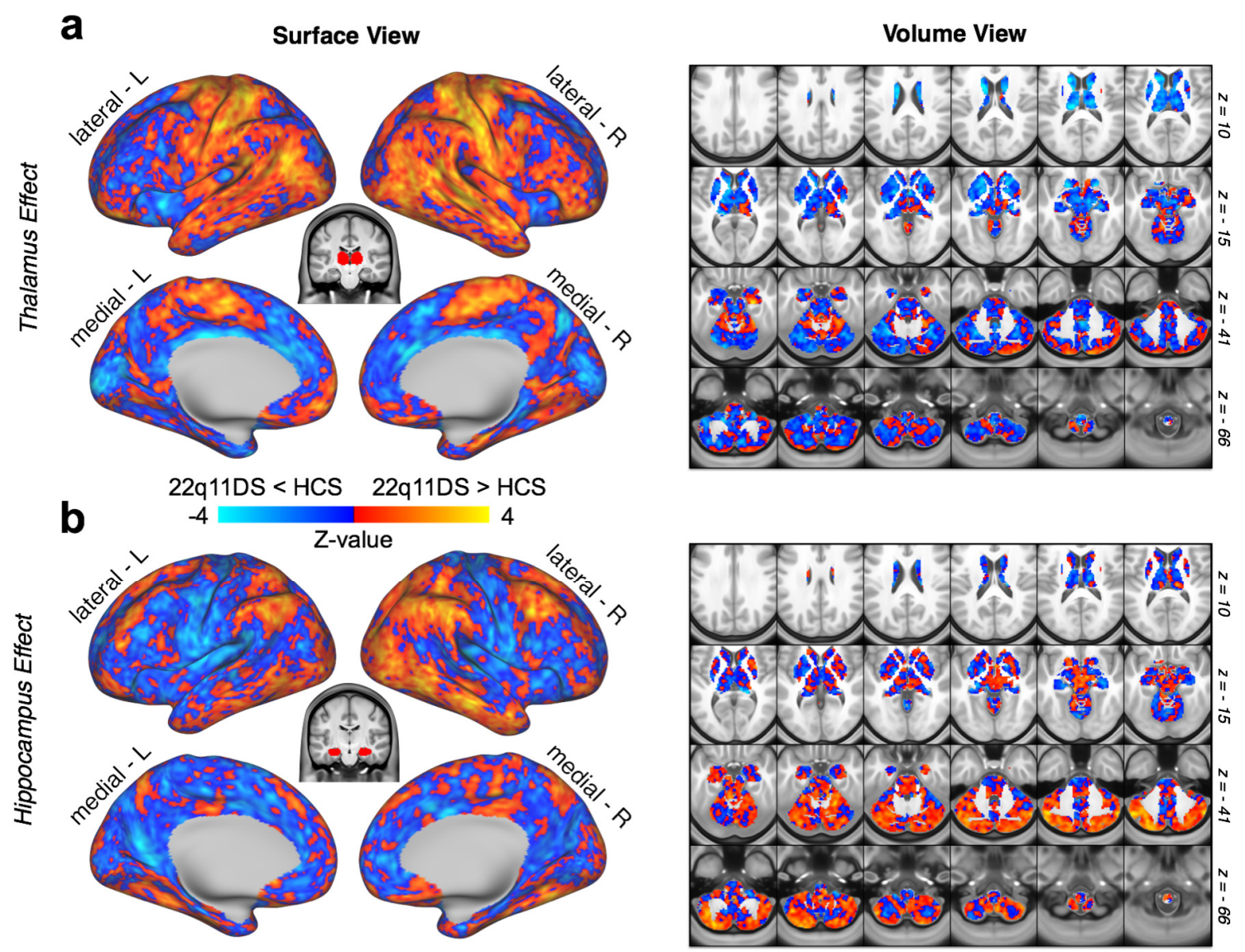

Figure 3. Threshold-Free Seed-Based Connectivity Maps. Threshold-free group contrasts for thalamic and hippocampal functional connectivity. (a) Surface and volume maps showing the threshold-free effect (22q11DS vs. HCS) for the thalamic seed. The yellow-orange contrast indicates 22q11DS $>$ HCS. Blue indicates HCS $>$ 22q11DS. (b) Same effect shown for the hippocampal seed.

Characterizing 22q11DS Dysconnectivity Across Thalamic and Hippocampal Sub-Regions. The thalamus and hippocampus are both heterogeneous structures which can be divided into multiple nuclei with distinct physiologies and connectivity profiles (Haber and McFarland, 2001). To assess differential functional connectivity disruptions across thalamic and hippocampal sub-regions, we used a k-means algorithm to cluster thalamic and hippocampal voxels based on unique between-group connectivity differences. The implementation of this algorithm is outlined in Figure 4. Figure 5 shows the k-means solutions for the thalamus and the hippocampus, both of which reveal distinct anterior and posterior clusters. The anterior thalamic cluster encompasses 'associative' thalamic nuclei (e.g. the medio-dorsal nucleus), whereas the posterior cluster is 
centered on visual lateral geniculate and pulvinar nuclei. The hippocampus was similarly divided along an anterior-posterior axis. Seed-based rs-fcMRI was subsequently computed for each thalamic and hippocampal cluster (group contrasts shown in Figure 5b). For both the thalamus and hippocampus, the whole-brain connectivity matrices for the anterior cluster were quantitatively more similar to the whole-seed effect (Figure 5c, whole-seed effects shown in Figure 3). For the thalamus specifically, due to its well-defined neuroanatomical subdivisions in humans, we also investigated how the cluster and whole-seed effects compared to the functional connectivity profiles of seven seeds derived from an FSL diffusion-weighted imaging thalamic atlas (Figure 6) (Behrens et al., 2003). As expected, both the anterior thalamic cluster and the whole-thalamus effects were most similar to a set of 'associative' thalamic seeds (prefrontal, temporal, premotor). In contrast, the posterior cluster effect was most similar to a set of 'sensory' thalamic seeds (occipital, sensory, parietal) (see Figure 6b).

\section{Illustration of Clustering Method \& Workflow}

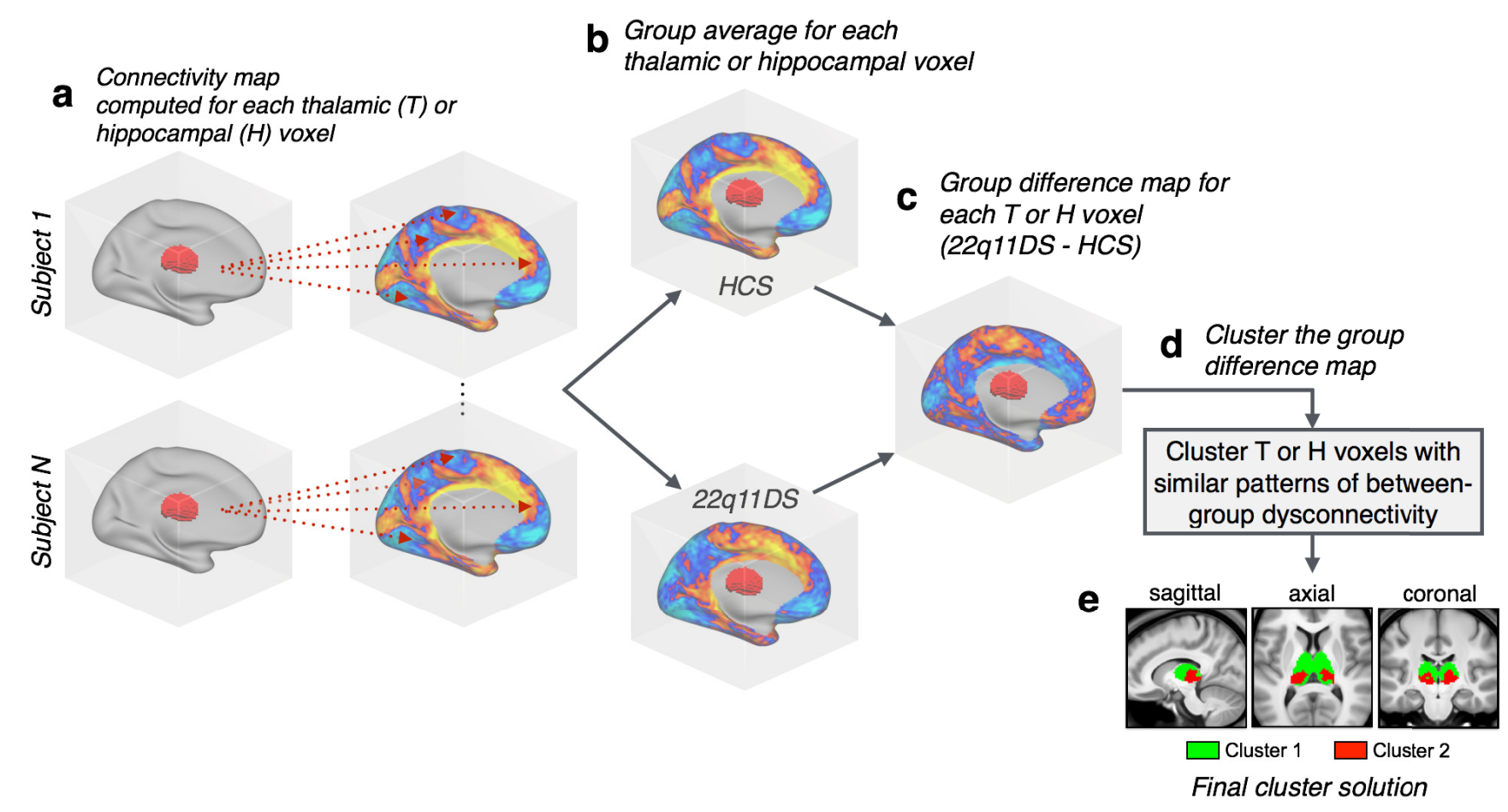

Figure 4. Clustering Algorithm Flow-Chart. A graphical illustration of the procedure for k-means clustering of thalamic voxels. This same procedure was repeated for the hippocampus. 
Main Text

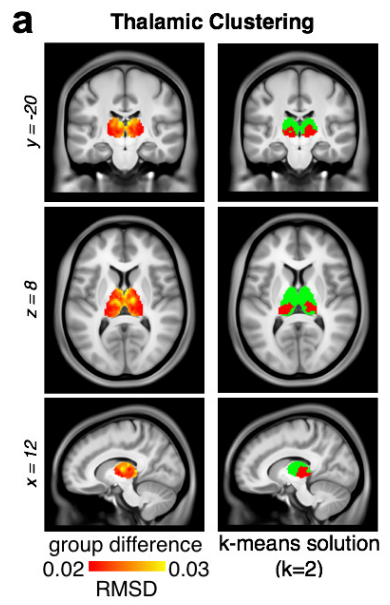

b
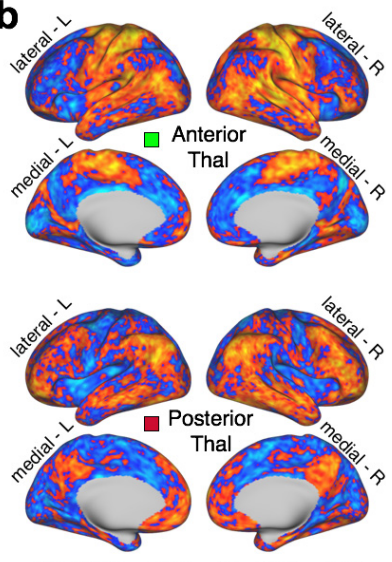

$\underset{-4}{22 q 11 D S}<$ HCS 22q11DS $>$ HCS

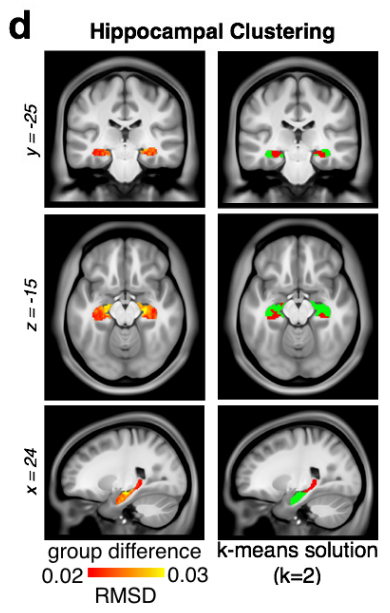

e
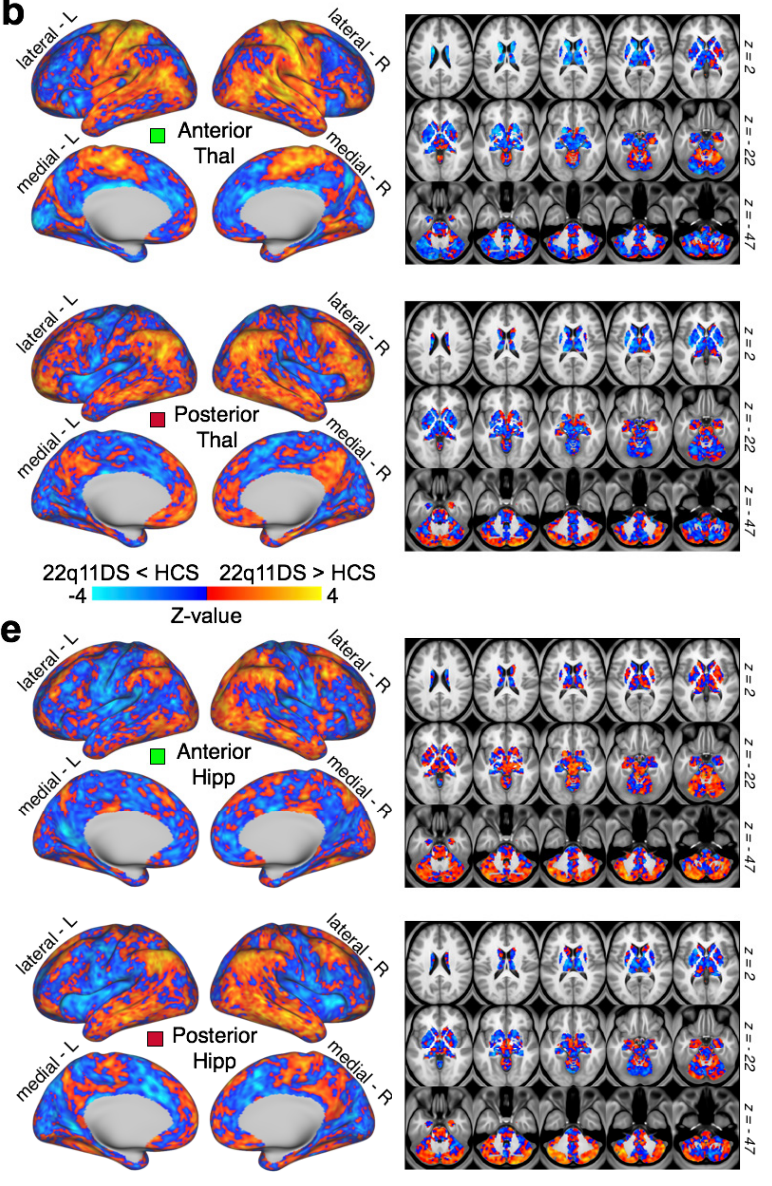

C
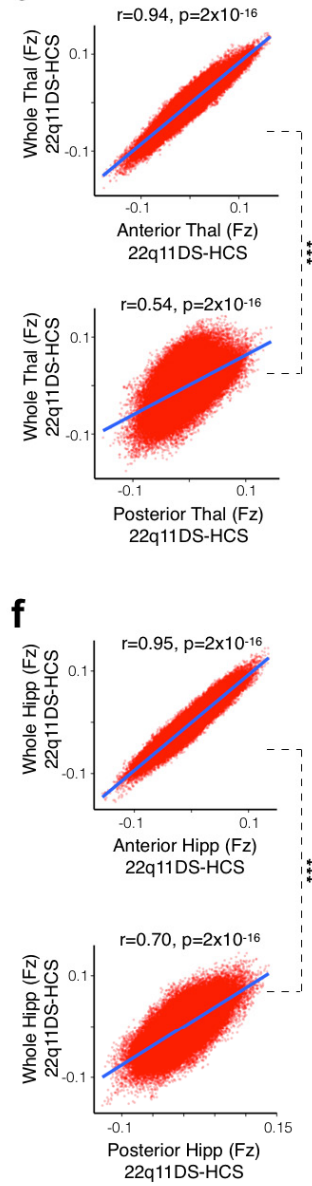

g

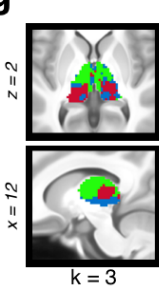

Thalamic Clustering $(k>2)$
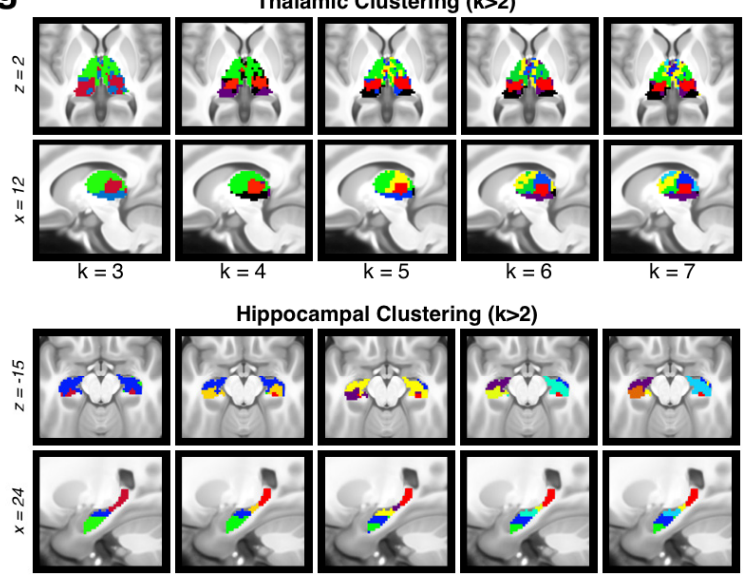

Hippocampal Clustering $(k>2)$
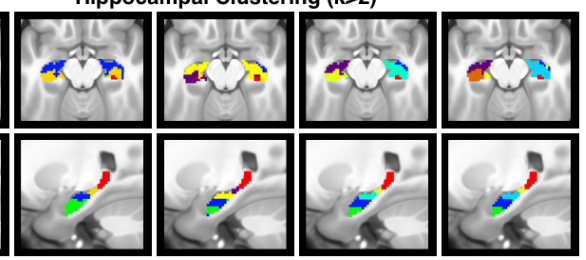

$\mathbf{h}$

Comparison of Cluster Solutions

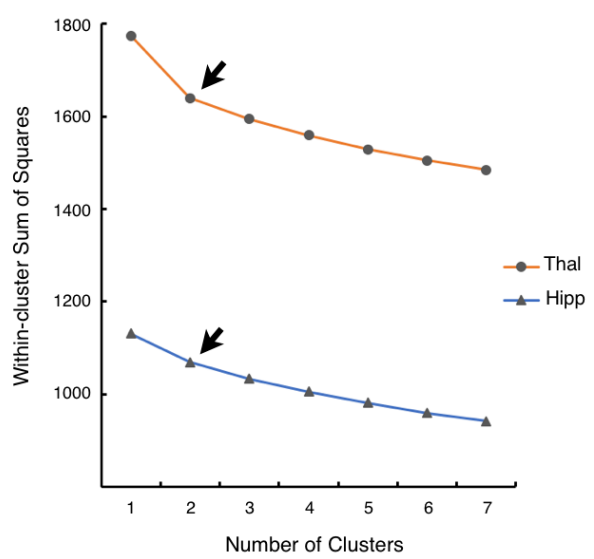

Figure 5. Data-Driven Clustering of Thalamic and Hippocampal Voxels. K-Means clustering of thalamus and hippocampus by group. (a) (left) Group difference map (root mean square) for the thalamus, highlighting the anterior and medial dorsal nuclei; (right) the $\mathrm{k}=2$ solution splits the thalamus into an anterior and posterior cluster. (b) Surface and volume maps show the group difference (22q11DS v. HCS) for the brain-wide functional connectivity of the anterior thalamic cluster (top), and posterior cluster (bottom). The yellow-orange contrast indicates 22q11DS $>$ HCS. 
Blue indicates HCS $>22$ q11DS. (c) Voxel-wise relationship between the brain-wide functional connectivity maps for each thalamic cluster versus the whole-thalamus seed (see Figure 3). The correlation with the whole-seed effect is significantly larger for the anterior cluster (top) compared to the posterior cluster (bottom) (Steiger's $\mathrm{z}=263, \mathrm{p}=2 \times 10^{-16}$ ). (d-f) Replication of (a-c) for the hippocampus, showing a similar anterior/posterior distinction $\left(\mathrm{z}=240, \mathrm{p}=2 \times 10^{-16}\right)$. (g) Distinct Kmeans solutions ranging from $\mathrm{K}=2$ to $\mathrm{K}=7$ always reveal an anterior solution (green). (h) Elbow plot illustrating percent variance explained by each progressive cluster solution.

a

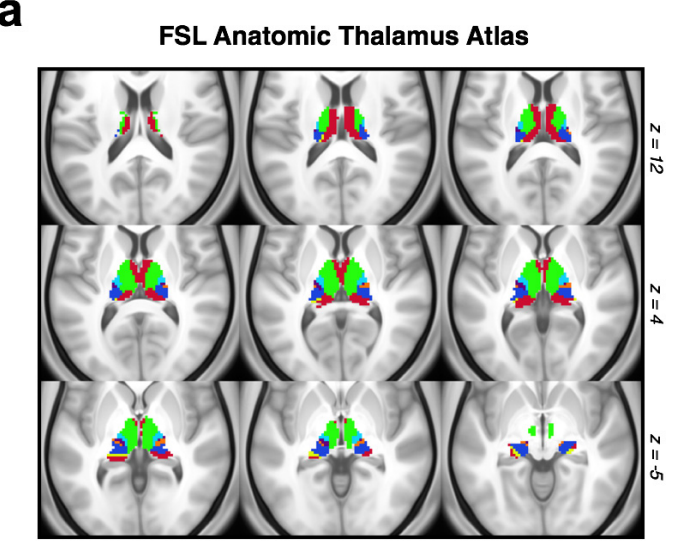

b

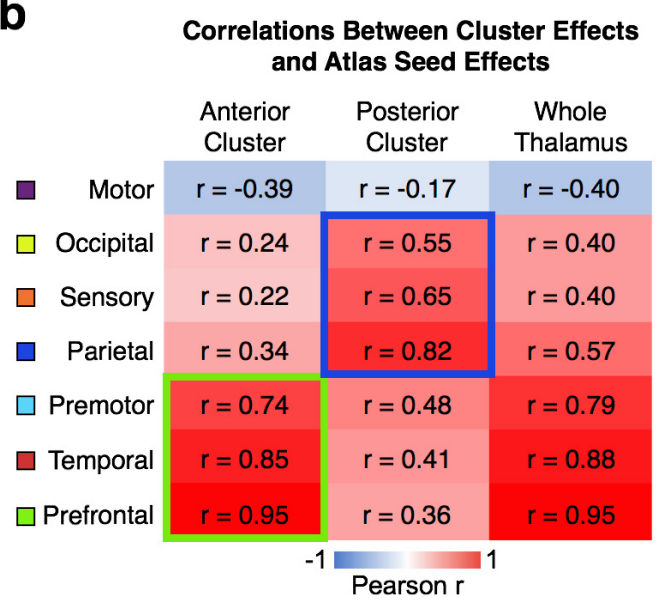

C

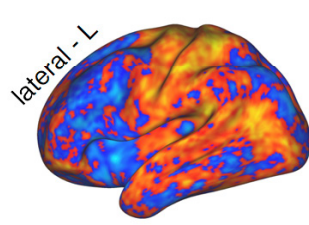

Thalamic a priori Anatomical Prefrontal Seed
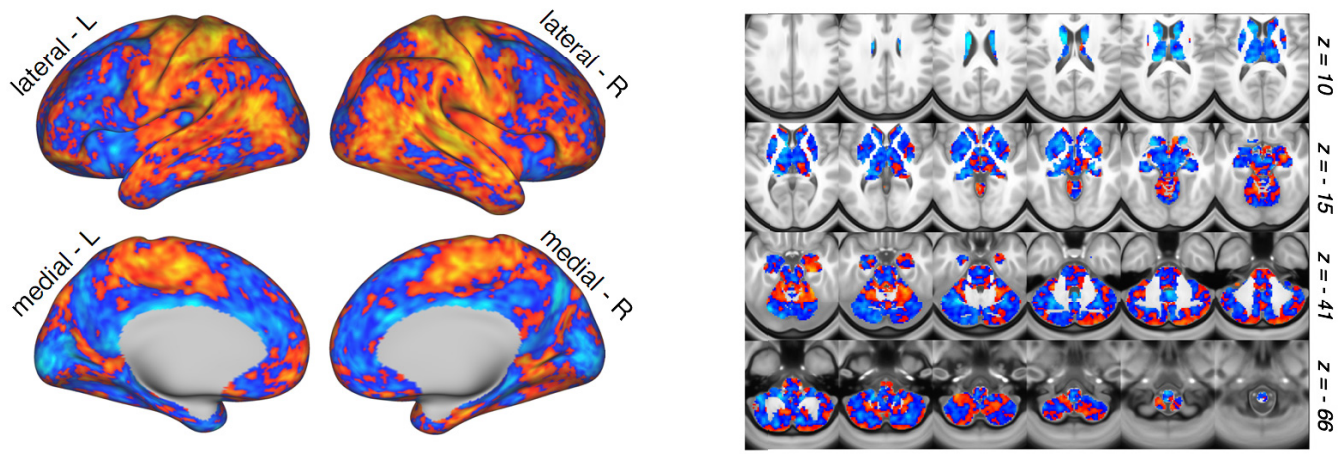

d

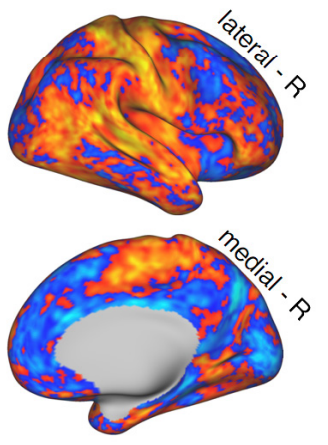

$22 q 11 D S<$ HCS 22q11DS $>$ HCS
$-4 \quad 4$

d Thalamic a priori Anatomical Parietal Seed
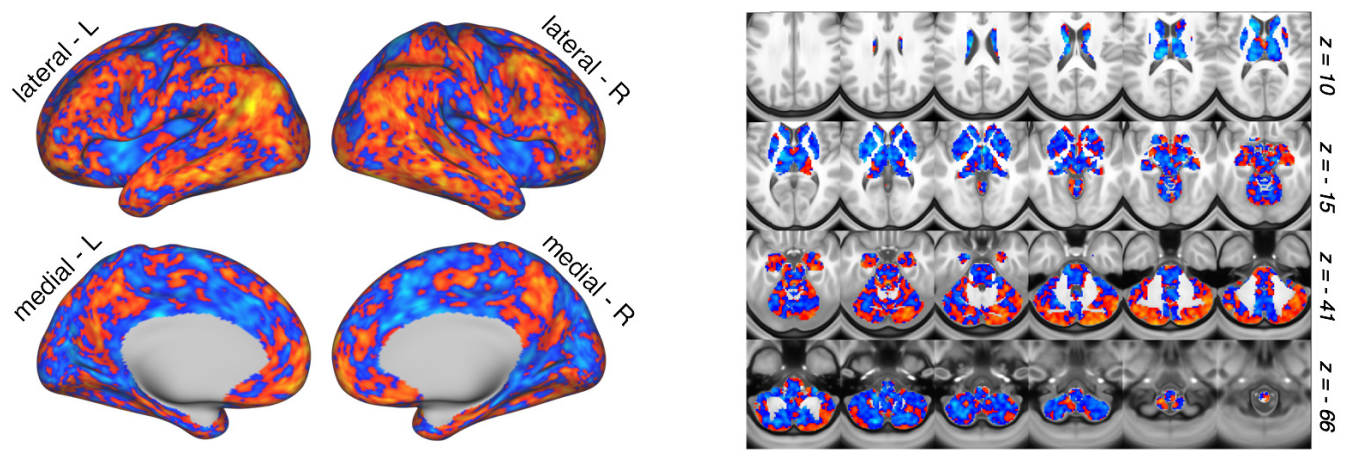

Figure 6. Quantifying Convergence Between K-means Solution and Independent Anatomicallydefined Thalamic Seeds. (a) FSL anatomic atlas of the thalamus derived from diffusion-weighted 
imaging. (b) Relationship between whole-brain maps of group differences derived from FSL's atlas and the $\mathrm{K}=2 \mathrm{~K}$-means solution, indicating a strong correspondence between the 'anterior' cluster and executive thalamic nuclei. (c) Thalamic a priori anatomically-defined prefrontal-projecting thalamic seed used to compute between-group differences, which matches the anterior $\mathrm{K}=2$ effect. (c) Thalamic a priori anatomically-defined parietal-projecting thalamic seed used to compute betweengroup differences, which matches the posterior $\mathrm{K}=2$ effect.

Effects of Global Signal Regression (GSR) on 22q11DS Dysconnectivity Profiles. As noted, prior to the main rs-fcMRI analyses, BOLD data were 'de-noised' via mean global signal regression (GSR), in order to attenuate the contribution from spatially pervasive sources of artifact, such as fluctuations in the magnetic field and non-neural physiological processes such as respiration (Power et al., 2017). Nevertheless, there is ongoing development regarding the best-practices for GSR in situations involving clinical populations (Glasser et al., 2017). To test if core observed rs-fcMRI effects are robust to GSR, we re-computed the main analyses without applying GSR. Notably, whole-brain thalamic and hippocampal functional connectivity maps were highly correlated pre- and post-GSR. Furthermore, the pre-GSR data extracted from the original interaction-derived ROIs (see Figure 2) showed the same interactive thalamic and hippocampal effects between groups. Finally, the type I error-corrected map for the pre-GSR results (as shown in Figure 7) fully overlapped with the original Figure 2 mask, but with somewhat greater spatial extent (for a detailed list of regions see Table 5). As such, results appear robust to GSR. 


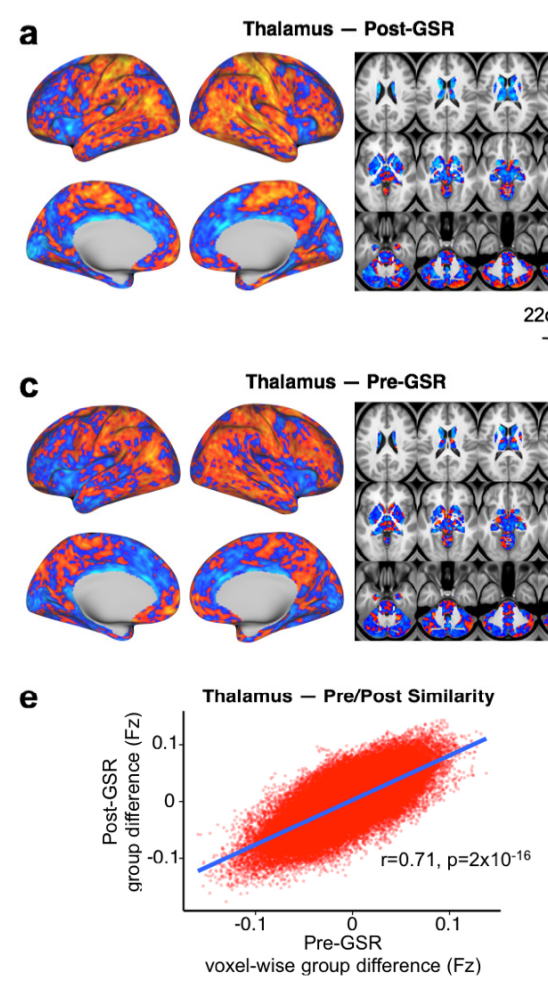

g

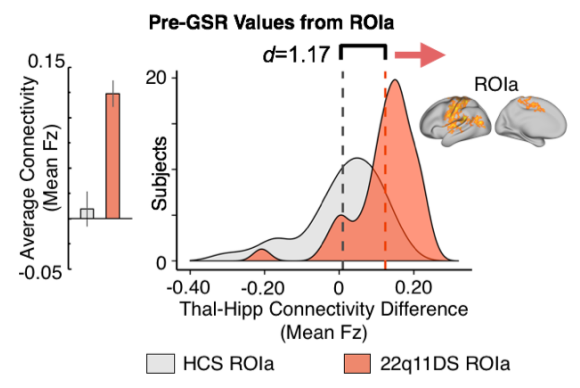

h

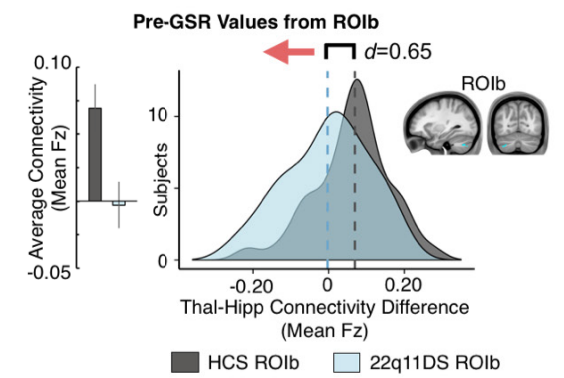

$\square$ HCS ROlb $\square$ 22q11DS ROIb

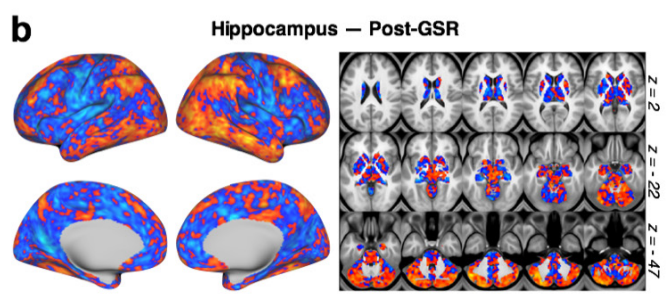

d

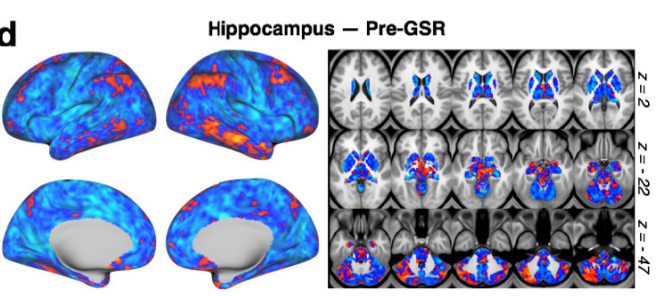

f
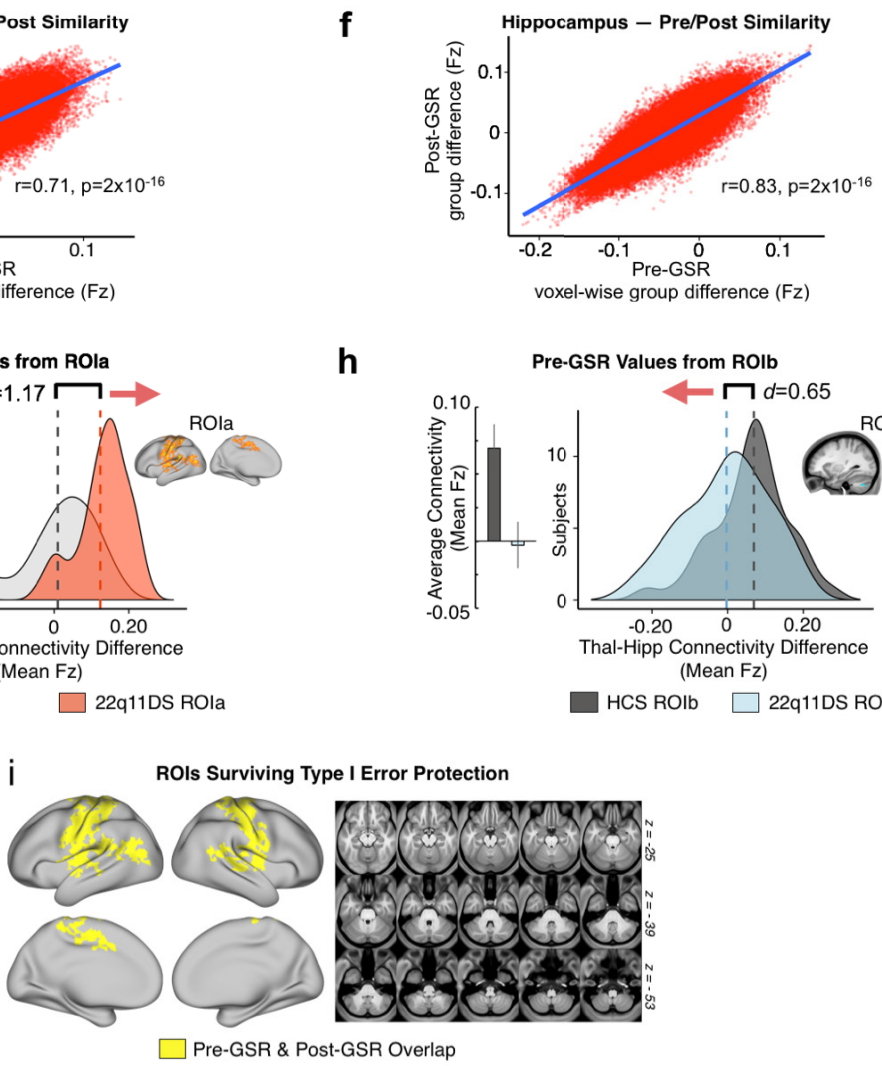

Figure 7. Stability of Effects Before and After Global Signal Regression. Here we show a comparison of pre- and post-GSR effects. (a-b) Post-GSR threshold-free connectivity for thalamus and hippocampus (same as Figure 3). (c-d) Thalamic and hippocampal connectivity before GSR. (e-f) Pearson correlation between pre- and post-GSR matrices. (g-h) pre-GSR data extracted from ROIa and ROIb (see Figure 2). (i) Overlapping regions (logical AND) for type I error-corrected interaction effect pre-GSR and post-GSR. The cerebellar effect (Figure 2 ROIb) did not survive without GSR. 
Interactive 22q11DS Disruptions across Sensory and Executive Networks. As noted, while we observed a focused type I error corrected effect in the cerebellum, the interactive results appeared substantially more widespread. Therefore, we tested whether 22q11DS patients indeed exhibit a network-level dissociation for thalamic versus hippocampal connectivity. To this end, we repeated the seed-based analyses focusing on thalamic and hippocampal connectivity to a priori networks derived from a data-driven parcellation of the human cortex, cerebellum, and striatum (Buckner et al., 2011; Choi et al., 2012; Yeo et al., 2011) (Figure 8). Here, functional connectivity was computed between the thalamic and hippocampal seeds and each of the seven a priori networks. In other words, we examined the connectivity between the thalamus or hippocampus with the entire brain-wide average of each functional network, yielding 14 values (i.e. 7 thalamus-to-each-network and 7 hippocampus-to-each-network rs-fcMRI values). As predicted, the 22q11DS group exhibited significantly increased thalamic but decreased hippocampal connectivity to brain-wide somatomotor (SOM) network regions, while the opposite effect was observed for the brain-wide frontoparietal (FPN) network regions.

Critically, across subjects, for both the interaction-derived and a priori network-derived effects, the magnitude of the rs-fcMRI effect in the sensory ROI/network was inversely related to the magnitude of the effect in the associative ROI/network (Figures 9 and 10). We quantified this relationship via Pearson correlations between the effects defined in the data-driven interaction-derived ROIs and the $a$ priori networks (SOM and FPN). 


\section{a}
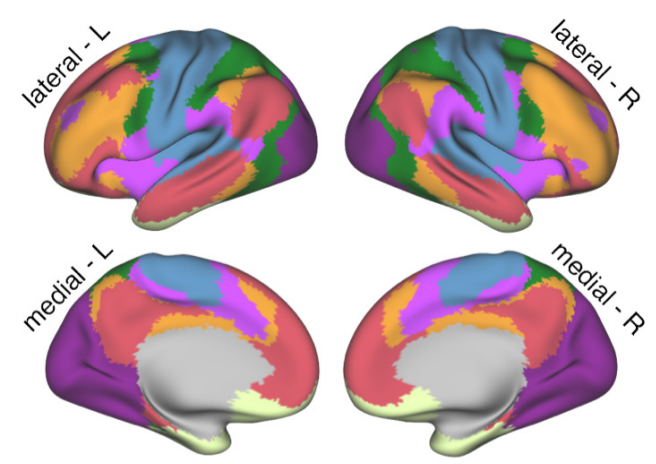

Volume View

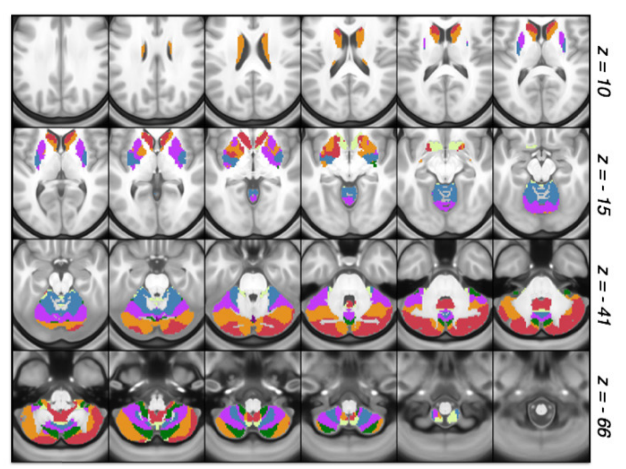

b
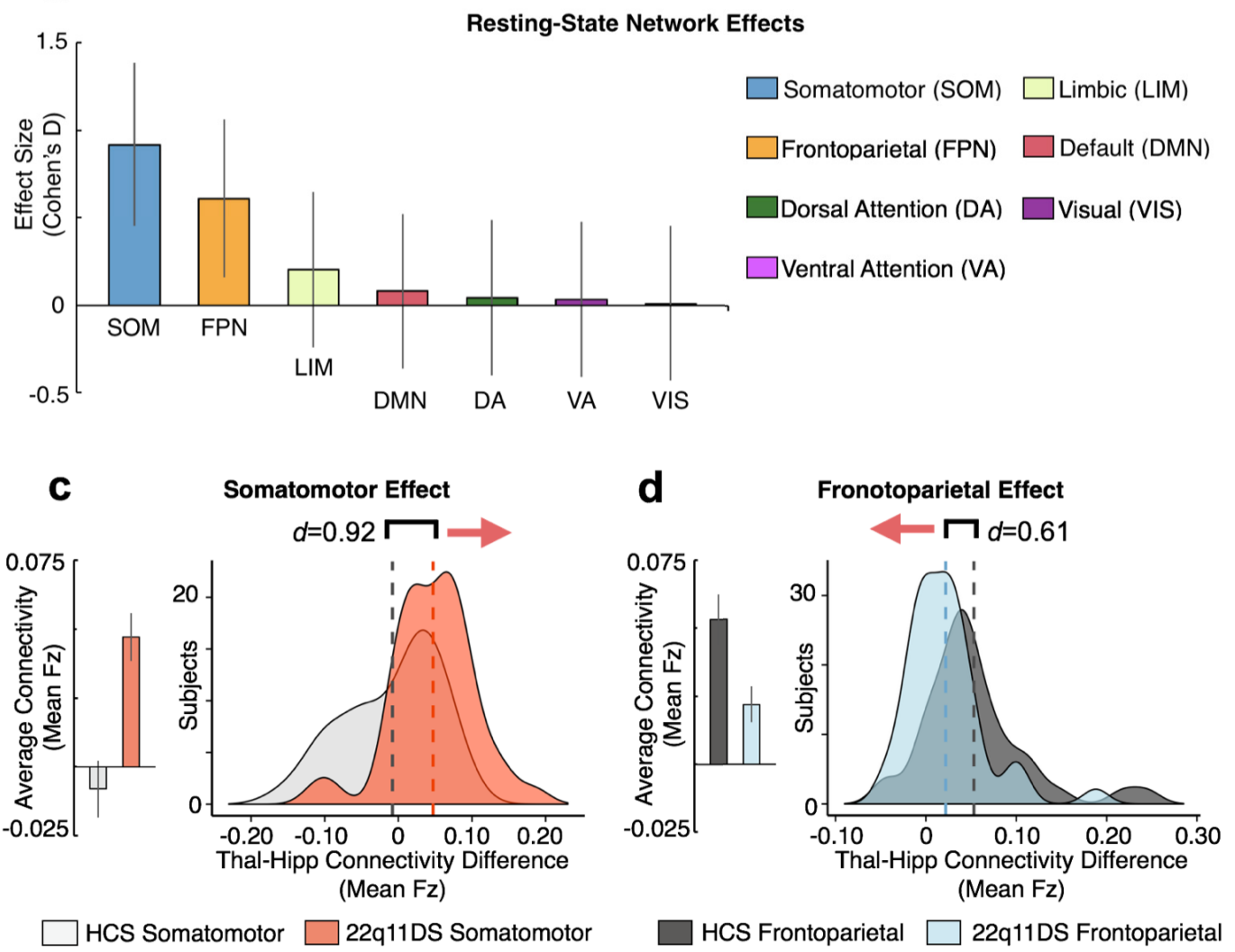

Figure 8. Examining Effects Across a priori Functional Networks. Replication of seed-based analysis (Figure 2) using a priori functionally-derived networks, mapped into CIFTI space (a). Surface and volume components of the map showing seven distinct functional networks derived from prior work that parcellated the cortex, striatum and cerebellum. Colors indicate distinct functional networks, following the same labeling pattern as the original work. (b) Effect sizes (Cohen's D) with 95\% confidence intervals comparing 22q11DS and HCS groups with regard to the difference scores between thalamic and hippocampal connectivity to each of the seven networks. (c) Thalamus-hippocampus difference scores illustrated for the somatomotor network (SOM) across subjects in each group. Group means (left) and distributions (right) illustrate the direction of the effect. (d) Same as (c) for the frontoparietal network (FPN), showing an effect in the opposite direction as SOM. 
a

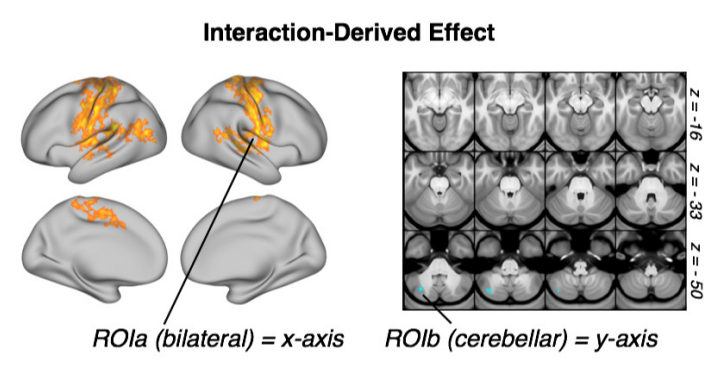

\section{C}

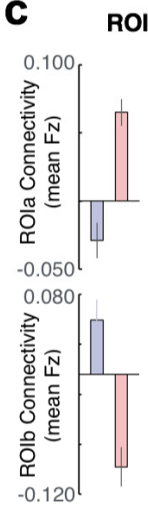

ROla v. ROIb: Thal-Hipp Connectivity Difference Scores

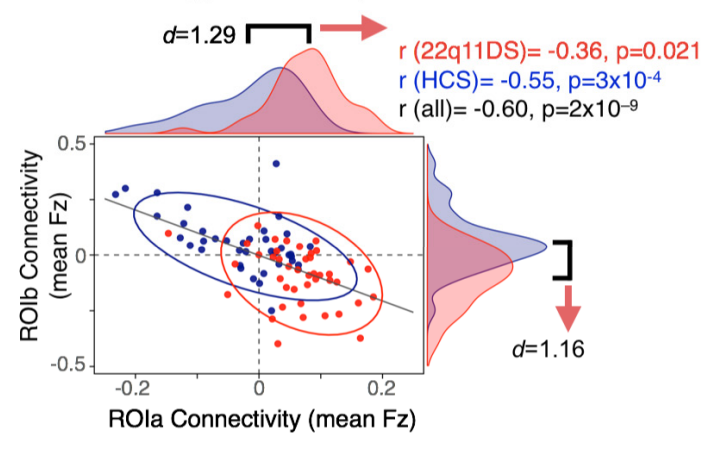

b

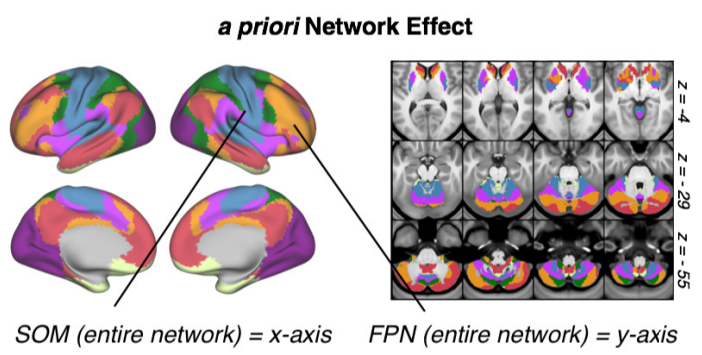

d

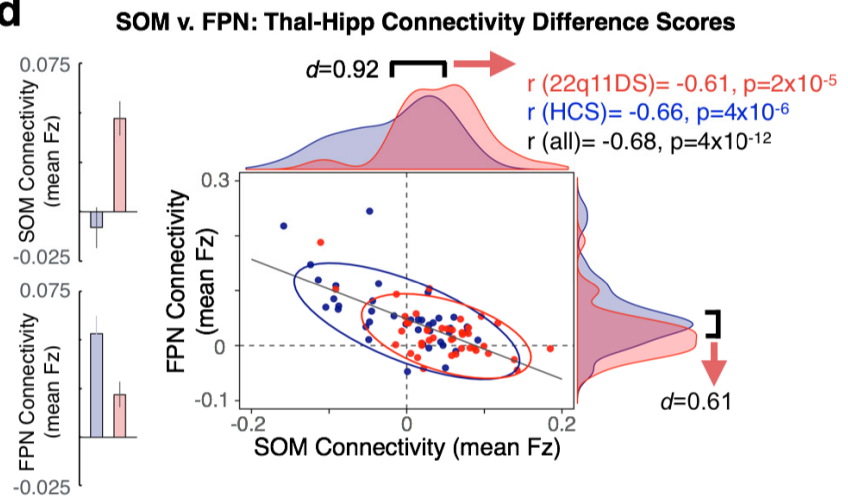

Figure 9. Relationship Between Regions of Reciprocally Disrupted Connectivity. (a) Across subjects, ROIa and ROIb (see Figure 2) are significantly negatively related in terms of the overall connectivity effect (thalamus-hippocampus $\mathrm{Fz}$ difference score). The distribution of 22q11DS subjects (red) is distinctly shifted relative to controls (blue), showing greater thalamic connectivity relative to hippocampal connectivity for ROIa, and the inverse for ROIb. (b) Replication of (a) using connectivity to a priori somatomotor and frontoparietal networks (see Figure 8), demonstrating that these reciprocal effects map onto large-scale sensory and associative networks. 22q11DS subjects show increased thalamic and decreased hippocampal connectivity to sensory regions, but the opposite effect in associative regions. Note: effects are presented for each seed and ROI pair individually in Figure 10. 
a
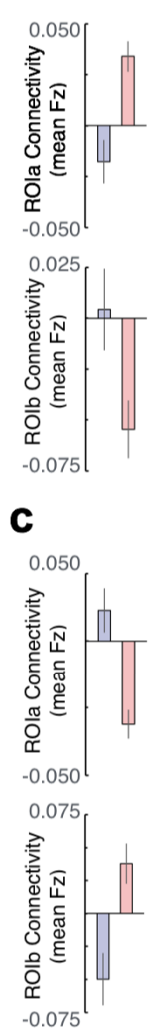

ROla v. ROlb: Thalamic Connectivity

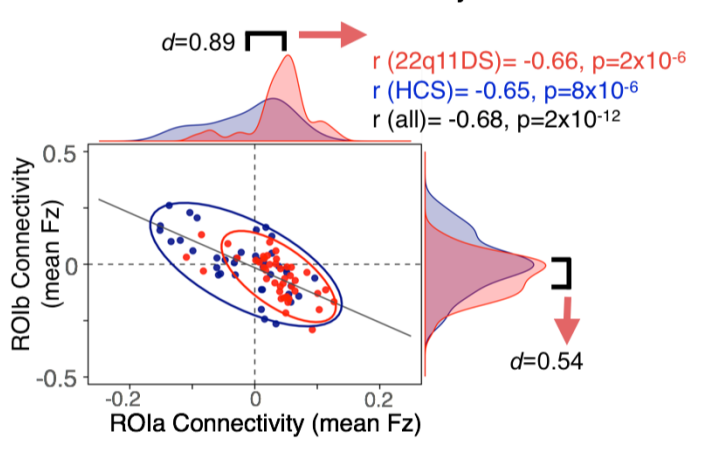

ROla v. ROIb: Hippocampal Connectivity

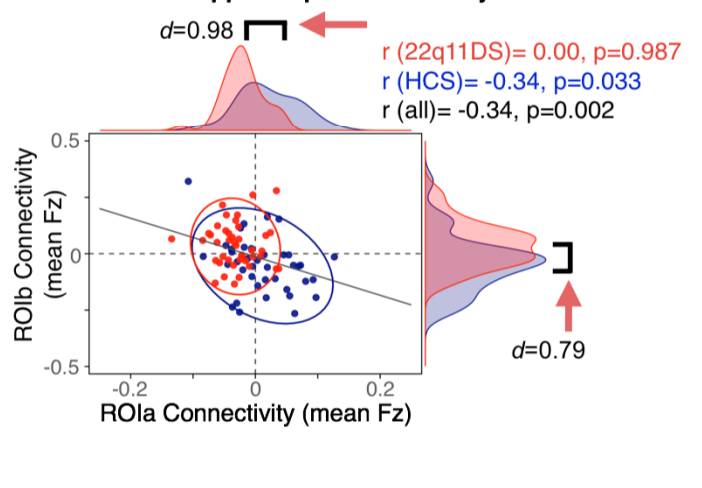

b

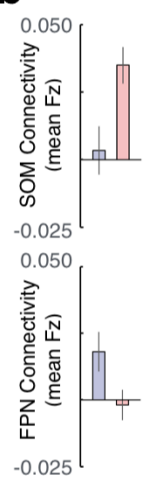

d

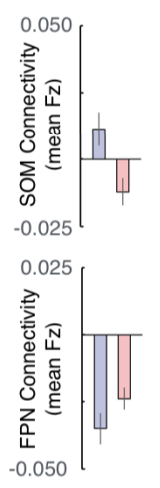

SOM v. FPN: Thalamic Connectivity

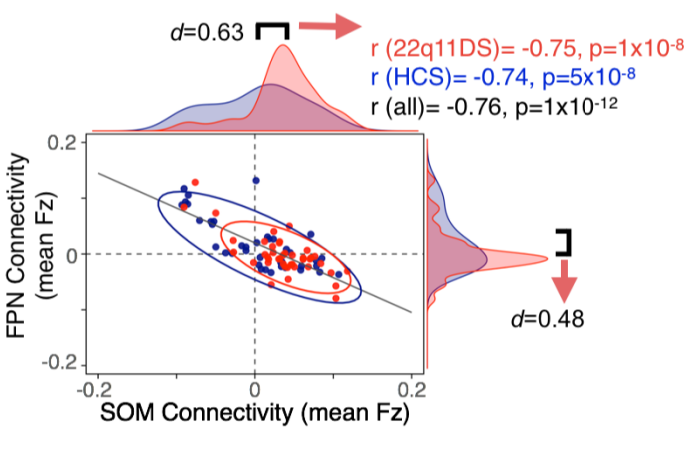

SOM v. FPN: Hippocampal Connectivity

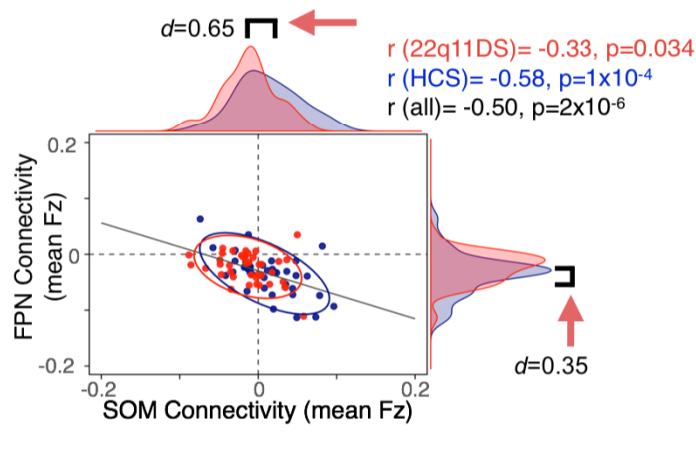

Figure 10. Reciprocal Effects Across Thalamic and Hippocampal Seeds. Expanding on Figure 9, showing distributions and relationships across subjects for whole thalamic and hippocampal seeds individually. The thalamic effect shifts in the opposite direction to the hippocampal effect.

\section{Prediction of 22q11 Case-Control Status from Data-driven and Network-level Dysconnectivity Effects. To}

test the hypothesis that the observed rs-fcMRI effects have potential utility as a neural biomarker, we conducted a SVM analysis (Figure 11). One-dimensional SVMs, computed based on the unweighted linear combination of thalamic and hippocampal connectivity to ROIa and ROIb (interaction-derived ROIs) correctly predicted diagnosis at rates well above chance (for $\mathrm{n}=1000$ iterations, mean $\mathrm{AUC}=0.843, \mathrm{SD}=0.043$ ). The unweighted combination of thalamic and hippocampal connectivity to entire a priori SOM and FPN networks was also able to provide moderate diagnostic accuracy (for $\mathrm{n}=1000$ iterations, mean $\mathrm{AUC}=0.739, \mathrm{SD}=0.057$ ). The fourdimensional SVM solution (i.e. combing the four discovered features: thal-ROIa, thal-ROIb, hipp-ROIa, hippROIb), which separated the groups by attempting to fit a hyper-plane that optimally weighted each factor 
(thalamic and hippocampal connectivity to each ROI/network), provided no performance advantage relative to the unweighted combination of features.
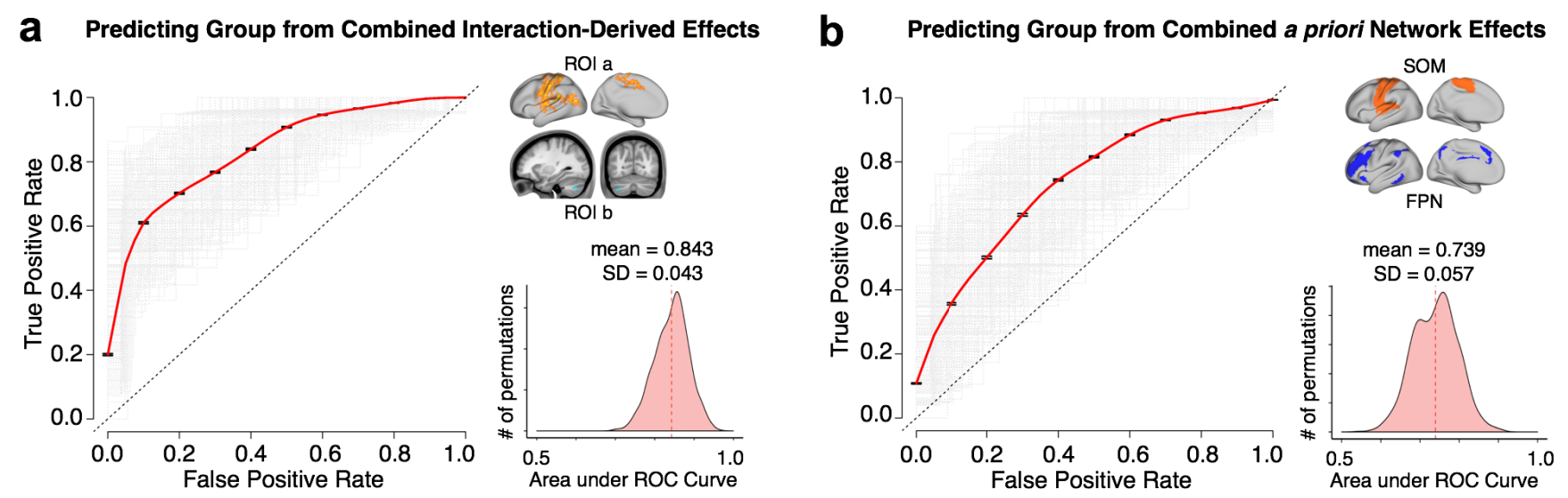

Figure 11. Diagnostic Classification via Support Vector Machine. SVM classification based on combined fc-MRI effects. (a) ROC curves for a binary classifier predicting group membership (22q11DS or HCS) based on the linear combination of connectivity values (mean Fz) from the thalamus and hippocampus to ROIa and ROIb ([thalROIa + hippROIb] - [thalROIb + hippROIa]). ROC curves for each of the $n=1000$ iterations are plotted in gray, and the vertical average in red. The distribution of areas under the $n=1000$ ROC curves (AUC) is plotted on the right, where an AUC of 1 would represent a perfect classifier. (b) replication of (a) using the linear combination of thalamic and hippocampal connectivity to somatomotor and frontoparietal networks ([thalSOM + hippFPN] - [thalFPN + hippSOM]). 


\section{DISCUSSION}

22q11DS is associated with notable neural alterations and presents a compelling genetic high-risk model in which anomalous circuitry can be investigated prior to development of overt psychiatric illness. Yet, there is a knowledge gap in our understanding of translational neural phenotypes in a genetic risk model such as 22q11DS. The thalamo-cortical system presents a unique leverage point for investigations of brain-wide dysconnectivity given its central locus and key role in functional and structural loops across the brain (Behrens et al., 2003; Zhang et al., 2010). Similarly, the hippocampus exhibits distinct brain-wide rs-fcMRI patterns relative to the thalamus in healthy humans (Figure 1), and structural and functional hippocampal alterations feature prominently across the neuropsychiatric spectrum (Tamminga et al., 2010). Notably, disruptions of this circuitry have been identified in a mouse model of the 22q11.2 deletion (Sigurdsson et al., 2010; Chun et al., 2014). The present study, for the first time, identified opposing patterns of thalamic-hippocampal disruption in human 22q11.2 deletion carriers. Specifically, findings revealed a pattern of significant thalamic overconnectivity with bilateral sensorimotor regions, including auditory cortex, in 22q11DS relative to typically developing controls, with the opposite effect in cerebellar regions. These findings extend prior work by identifying reciprocal and functionally linked disruptions of hippocampal connectivity in 22q11DS. This effect was verified via a priori networks derived from a data-driven parcellation of the human cortex, cerebellum, and striatum (Buckner et al., 2011; Yeo et al., 2011; Choi et al., 2012). Again, the 22q11DS group showed an inverse pattern relative to controls, with significantly increased thalamic and decreased hippocampal connectivity to SOM regions, and the opposite effect in FPN networks. Data-driven k-means clustering showed, for the first time, that the anterior portions of thalamus and hippocampus were driving the observed patterns of disrupted connectivity. This result is in concert with the view that these are heterogeneous structures, which can be divided into multiple nuclei with distinct physiologies and connectivity profiles. Finally, machine learning analyses revealed accurate classification of 22q11DS patients versus controls, based on the un-weighted linear combination of thalamic and hippocampal connectivity at rates well above chance $(84 \%$ overall classification 
accuracy). These effects indicate potential utility of the reported thalamo-hippocampal dysconnectivity for prediction of future development of neuropsychiatric symptoms.

Implications for the Neurobiology of Psychosis. Thalamic over-connectivity with sensorimotor regions and cerebellar under-connectivity in 22q11DS are in line with prior observations in patients with established schizophrenia (Anticevic et al., 2014) and those at clinical high-risk for the disorder (Anticevic et al., 2015b). Notably, the reported thalamic effect was particularly prominent in those clinical high-risk youth who subsequently converted to psychosis, which would suggest that these network-level disturbances are present prior to onset of overt illness. Note that in 22q11DS, hypo-connectivity with broader executive regions was supported by network-level FPN analysis. Our findings of reciprocal thalamic-hippocampal effects are particularly notable, given that the nucleus reuniens of the thalamus directly innervates the hippocampus (Herkenham, 1978; Lisman, 2012), and was recently determined to play a key role in regulating bi-directional communication between the dorsal hippocampus and medial prefrontal cortex (Hallock et al., 2016). This hypothesis is further supported by the k-means solutions, which implicate the key functional roles of 'anterior' subdivisions for both thalamic and hippocampal seeds.

Nevertheless, BOLD rs-fMRI is an indirect observational neuroimaging measure, and thus cannot address underlying cellular mechanisms. However, these processes can be investigated in translational studies in animal (Hiroi et al., 2013), and in vitro models (Brennand et al., 2012) as well as computational modeling studies, which can generate testable predictions at the circuit level (Anticevic et al., 2015a). Theoretical models of psychosis implicate alterations in glutamatergic, dopaminergic and inhibitory GABAergic neurotransmission, which may be relevant to the observed disruptions of thalamo-striatal-cortical circuitry (Gonzalez-Burgos and Lewis, 2012; Lewis et al., 2012; Woodward et al., 2012). At present, the origin of the widespread reciprocal thalamic-hippocampal disruption is not fully understood. However, investigation of this circuitry in the context of a well-characterized genetic etiology, as demonstrated in the current study, is a key advantage and a path 
forward. One possibility may involve dysfunction of N-methyl-D-aspartate glutamate receptors (NMDAR) (Javitt, 2007; Loh et al., 2007), which may impact excitatory-inhibitory balance in cortical circuits and lead to large-scale disturbances in thalamo-cortical information flow. Notably, this hypothesis is supported by data from the 22q11.2 mouse model, as discussed below. Alternatively, it is possible that a local 'hotspot' of dysfunction (e.g. such as the nucleus reuniens of the thalamus) emerges, via confluence of polygenic risk (Anticevic and Lisman, 2017).

Convergence with 22q11.2 Mouse Model. In a mouse model of the 22q11.2 deletion, Chun and colleagues reported disrupted glutamatergic synaptic transmission at thalamic inputs to the auditory cortex (Chun et al., 2014), suggesting that thalamo-cortical disruption could be a pathogenic mechanism that mediates susceptibility to positive psychotic symptoms in 22q11DS. Furthermore, it was determined that thalamo-cortical disruption in 22q11DS mice was caused by abnormal elevation of dopamine D2 (DRD2) receptors in the thalamus. Increased DRD2 in the thalamus and other brain regions has been reported in antipsychotic naïve schizophrenia patients (Oke et al., 1988; Cronenwett and Csernansky, 2010). The dgcr8 gene, which encodes part of the microprocessor complex that mediates microRNA (miRNA) biogenesis, was pinpointed as being responsible for this neuronal phenotype in the 22q11DS mouse model. Consequently, reduced dosage of $d g c r 8$ in $22 \mathrm{q} 11 \mathrm{DS}$ may lead to miRNA dysregulation, and downstream disruption of synaptic function and proper neural circuit development (Earls and Zakharenko, 2014).

More recently, Chun et al. further established a thalamus-enriched miRNA in the 22q11DS mouse model, which specifically targets DRD2 (miRNA 338-3p). This may be a key mediator of the disruption of synaptic transmission at thalamo-cortical projections and the late adolescent/early adult onset of auditory perceptual anomalies in individuals with 22q11DS (Chun et al., 2016).

Although, to our knowledge, reciprocal disruption of the hippocampal-thalamic circuit has not yet been directly probed in this mouse model, there is complementary evidence for impaired synchronization of neural 
activity between the hippocampus and prefrontal cortex. Specifically, Sigurdsson and colleagues (2010) found that, while hippocampal-prefrontal synchrony increased during working memory performance in wild-type mice, this phase-locking did not occur in the 22q11DS mice. Further, the magnitude of baseline hippocampalprefrontal coherence was predictive of how long it took the mice to learn the task. Taken together, these findings suggest that observations of disrupted large-scale network coherence in human 22 q11.2 deletion carriers are recapitulated in the animal model. Recent studies from rodent models have also revealed a broader role of the thalamus in higher-order cognitive functions such as working memory. In fact, working memory maintenance required mediodorsal thalamic inputs, suggesting a causal role of dysfunction in this circuit in characteristic cognitive deficits associated with schizophrenia (Bolkan et al., 2017).

Pitfalls and Future Solutions. Notably, only a minority of 22q11DS participants were taking medications at the time of the scan, and thus it is unlikely that medication effects played a role in the observed findings. Another concern, present across rs-fcMRI studies in clinical populations, relates to head movement. We movement-scrubbed all data and used movement (\% frames scrubbed) as a covariate across all analysis, which did not alter the observed findings. Furthermore, motion parameters did not significantly differ between 22q11DS and typically developing control participants (Table 1) and rs-fcMRI effects were not related to head movement or signal-to-noise ratio (SNR) (Table 2). Finally, we studied subjects who, by virtue of a highly penetrant $\mathrm{CNV}$, were at elevated risk for psychosis (and other neuropsychiatric symptoms). Given the young age of many of the study participants, current findings cannot address the question of whether the magnitude of thalamic-hippocampal dysconnectivity is indeed associated with subsequent risk for the development of psychosis. Importantly, the classification results indicate robust sensitivity-specificity, which may aid such prediction. Prospective longitudinal studies are currently underway to address this key knowledge gap. 
Conclusions. This study leverages the well-characterized genetic etiology of 22q11DS, thus providing a robust high-penetrance model to guide and test mechanistic hypotheses regarding disrupted brain development and subsequent consequences for circuit dysfunction leading to neuropsychiatric symptoms. Our findings offer the first evidence for reciprocal disruption of thalamic and hippocampal functional connectivity with cortical regions in this genetic risk model. Notably, the observed findings pinpoint an anterior axis of thalamichippocampal systems in line with animal model observations, which yield a robust classifier that can be refined for longitudinal risk prediction. These findings suggest that ongoing focus on thalamic-hippocampal circuit interactions in 22q11DS patients and in animal models can guide translational development of targeted and mechanistically informed neural markers and subsequent therapeutics. 
bioRxiv preprint doi: https://doi.org/10.1101/226951; this version posted November 29, 2017. The copyright holder for this preprint (which was not certified by peer review) is the author/funder, who has granted bioRxiv a license to display the preprint in perpetuity. It is made available under aCC-BY-ND 4.0 International license.

\begin{tabular}{|c|c|c|c|c|c|c|c|c|c|c|c|c|}
\hline $\mathrm{X}$ & $\mathrm{Y}$ & 7 & & & \multicolumn{8}{|c|}{ le 4. Pairwise Comparisons - Region Coordinates, P-values \& Effect Size } \\
\hline & & & & & & & & & & & & \\
\hline-5 & 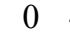 & 43 & corte & sulate $\mathrm{g}$ & $348 \mathrm{~mm} 2$ & Pt-Con Thal. 0.24 & -1.09 & 0.279 & Pt-Con Hipp. & 0.58 & 2.62 & .010 \\
\hline-13 & -37 & 75 & cortex left & postcentral gyrus & $76 \mathrm{mn}$ & Pt-Con Thal. 0.45 & -2.01 & 0.048 & Pt-Con Hipp. & 0.40 & 1.82 & 0.073 \\
\hline-8 & -35 & 60 & cortex left & paracentral lobule & $68 \mathrm{~mm} 2$ & Pt-Con Thal. 0.58 & -2.59 & 0.011 & Pt-Con Hipp. & 0.27 & 1.22 & 0.225 \\
\hline-9 & -26 & 51 & cortex left & cingulate sulcus & $304 \mathrm{~mm} 2$ & Pt-Con Thal. 0.36 & -1.61 & 0.111 & Pt-Con Hipp. & 0.48 & 2.14 & 0.036 \\
\hline-6 & -26 & 43 & cortex left & paracentral lobule & $268 \mathrm{~mm} 2$ & Pt-Con Thal. 0.51 & -2.29 & 0.025 & Pt-Con Hipp. & 0.18 & 0.80 & 0.424 \\
\hline-6 & -4 & 56 & cortex left & paracentral lobule & $372 \mathrm{~mm} 2$ & Pt-Con Thal. 0.60 & -2.69 & 0.009 & Pt-Con Hipp. & 0.29 & 1.32 & 0.189 \\
\hline-37 & -7 & 65 & cortex left & precentral sulcus & $220 \mathrm{~mm} 2$ & Pt-Con Thal. 0.50 & -2.23 & 0.029 & Pt-Con Hipp. & 0.32 & 1.44 & 0.154 \\
\hline-18 & -19 & 76 & cortex left & precentral gyrus & $420 n$ & Pt-Con Thal. 0.41 & -1.84 & 0.069 & Pt-Con Hipp. & 0.42 & 1.89 & 0.063 \\
\hline-16 & -5 & 74 & corte & uperior frontal gyrus & $404 \mathrm{~m}$ & Pt-Con Thal. 0.39 & -1.77 & 0.081 & Pt-Con Hipp. & 0.51 & 2.29 & 0.025 \\
\hline-58 & -18 & 46 & cortex left & postcentral gyrus & $684 \mathrm{~m}$ & Pt-Con Thal. 0.72 & -3.23 & 0.002 & Pt-Con Hipp. & 0.50 & 2.26 & 0.026 \\
\hline-39 & -43 & 62 & cortex left & superior parietal lobule & $944 \mathrm{~mm} 2$ & Pt-Con Thal. 0.92 & -4.15 & 0.000 & Pt-Con Hipp. & 0.17 & 0.78 & 0.436 \\
\hline-29 & -35 & 72 & cortex left & postcentral gyrus & $336 \mathrm{~mm} 2$ & Pt-Con Thal. 0.53 & -2.38 & 0.020 & a Hipp. & 0.32 & 1.46 & 0.148 \\
\hline-39 & -26 & 64 & cortex left & central sulcus & $592 \mathrm{~mm} 2$ & Pt-Con Thal. 0.52 & -2.35 & 0.021 & Pt-Con Hipp. & 0.60 & 2.72 & 0.008 \\
\hline-49 & -9 & 49 & cortex left & central sulcus & $556 \mathrm{~mm} 2$ & Pt-Con Thal. 0.33 & -1.49 & 0.140 & Pt-Con Hipp. & 0.73 & 3.27 & 0.002 \\
\hline-64 & -28 & 0 & cortex left & superior temporal gyrus & $200 \mathrm{~mm} 2$ & Pt-Con Thal. 0.33 & -1.48 & 0.143 & A Hipp. & 0.59 & 2.64 & 0.010 \\
\hline-55 & -39 & 22 & cortex left & posterior sy & $824 \mathrm{~m}$ & Pt-Con Thal. 0.61 & -2.76 & 0.007 & $\mathrm{Pt}$ & 0.76 & 3.43 & 0.001 \\
\hline$-61-$ & -26 & 6 & cortex left & medial temporal lobe & $408 \mathrm{~m}$ & Pt-Con Thal. 0.41 & -1.84 & 0.069 & $\mathrm{Pt}-$ & 0.77 & 3.46 & 0.001 \\
\hline-42 & -12 & 8 & cortex left & inculo & $560 \mathrm{r}$ & Pt-Con Th & -0.91 & 0.367 & & 0.97 & 4.34 & \\
\hline-46 & -86 & 3 & cortex left & inferior occipital gyrus & $92 \mathrm{~mm} 2$ & Pt-Con The & -2.82 & 0.006 & $\mathrm{Pt}-$ & 0.21 & 0.95 & 0.348 \\
\hline-49 & -77 & 13 & cortex left & superior occipital gyrus & 696 & Pt-Con Thal. 0.73 & -3.29 & 0.001 & & 0.27 & 1.24 & 0.220 \\
\hline-53 & -17 & 16 & cortex left & sylvian fissure & $728 \mathrm{~mm} 2$ & Pt-Con Thal. 0.26 & -1.16 & 0.250 & Pt-Con Hipp. & 0.81 & 3.63 & 0.001 \\
\hline-62 & -30 & 40 & cortex left & supramarginal gyrus & $348 \mathrm{~mm} 2$ & Pt-Con Thal. 0.54 & -2.44 & 0.017 & Hipp. & 0.27 & 1.24 & 0.220 \\
\hline-66 & -10 & 25 & corte & postce & 468 & 0.40 & -1.78 & 0.079 & $\mathrm{Pt}$ & 0.73 & 3.27 & 0.002 \\
\hline-60 & -7 & 33 & cort & cent & 53 & 0.39 & -1.75 & 0.084 & $\mathrm{Pt}$ & 0.63 & 2.81 & 0.006 \\
\hline-56 & 5 & 5 & cort & sylv & 3 & 0.31 & -1.40 & 0.165 & & 0.80 & 3.61 & 0.001 \\
\hline-52 & -10 & -5 & cort & edial t & 25 & Pt-Con Th & -1.93 & 0.057 & $\mathrm{Pt}$ & 0.72 & 3.26 & 0.002 \\
\hline-53 & 0 & -12 & cortex left & medial temr & 172 & Pt-Con Thal. 0.47 & -2.10 & 0.039 & Pt-Con Hipp. & 0.54 & 2.41 & 0.018 \\
\hline-61 & -10 & -2 & cortex left & superior temporal gyrus & $284 \mathrm{~mm} 2$ & Pt-Con Thal. 0.35 & -1.58 & 0.119 & Hipp. & 0.73 & 3.26 & 0.002 \\
\hline-62 & -32 & -9 & cortex left & superior temporal sulcus & $208 \mathrm{~mm} 2$ & Pt-Con Thal. 0.46 & -2.06 & 0.043 & Pt-Con Hipp. & 0.26 & 1.15 & 0.253 \\
\hline 9 & -25 & 72 & cortex right & paracen & $144 \mathrm{~mm} 2$ & Pt-Con Thal. 0.46 & -2.08 & 0.041 & $\mathrm{Pt}-\mathrm{C}$ & 0.28 & 1.25 & 0.215 \\
\hline 45 & -10 & 59 & corte & $x=0$ & & .65 & -2.91 & 0.005 & & 0.49 & 2.22 & 0.029 \\
\hline 18 & -34 & 76 & cort & postc & 26 & 0.48 & -2.15 & 0.035 & $\mathrm{Pt}$ & 0.42 & 1.89 & 0.062 \\
\hline 31 & -13 & 72 & corte & prec & $368 \mathrm{r}$ & 0.84 & -3.78 & 0.000 & $\mathrm{Pt}-$ & 0.19 & 0.84 & 0.401 \\
\hline 15 & -21 & 76 & corte & prece & 1 & Con Thal. 0.31 & -1.41 & 0.163 & $\mathrm{Pt}-\mathrm{C}$ & 0.55 & 2.49 & 0.015 \\
\hline 55 & -3 & 46 & corte & central sulcus & $164 \mathrm{~mm} 2$ & Pt-Con Thal. 0.20 & -0.88 & 0.380 & Pt-Con Hipp. & 0.52 & 2.32 & 0.023 \\
\hline 29 & -29 & 70 & cortex right & central sulcus & $440 \mathrm{~mm} 2$ & Pt-Con Thal. 0.39 & -1.75 & 0.083 & Pt-Con Hipp. & 0.74 & 3.32 & 0.001 \\
\hline 48 & -27 & 64 & cortex right & postcentral gyrus & $444 \mathrm{~mm} 2$ & Pt-Con Thal. 0.75 & -3.37 & 0.001 & Pt-Con Hipp. & 0.18 & 0.81 & 0.423 \\
\hline 45 & -21 & 59 & corte & centr & $624 \mathrm{~mm} 2$ & Pt-Con Thal. 0.59 & -2.68 & 0.009 & Pt-Con Hipp. & 0.62 & 2.78 & 0.007 \\
\hline 59 & -45 & 14 & corte & rior te & & 83 & -3.73 & 0. & $\mathrm{Pt}$ & 0.26 & 1.18 & 0.241 \\
\hline 65 & -34 & 6 & corte & & & 0.81 & -3.62 & 0.0 & Pt- & 0.46 & 2.05 & 0.044 \\
\hline 50 & -27 & 11 & corte & In fissure & 33 & 0.12 & -0.55 & 0.5 & Pt- & 0.89 & 4.00 & 0.000 \\
\hline 44 & -1 & 3 & cortex right & inst & $380 \mathrm{~mm} 2$ & Pt-Con Thal. 0.48 & -2.16 & 0.034 & Pt-Con Hipp. & 0.58 & 2.60 & 0.011 \\
\hline 20 & -44 & 75 & cortex right & postcentral sulcus & $444 \mathrm{~mm} 2$ & Pt-Con Thal. 0.56 & -2.50 & 0.014 & Pt-Con Hipp. & 0.45 & 2.04 & 0.044 \\
\hline 31 & -50 & 70 & cortex right & superior parietal lobule & $360 \mathrm{~mm} 2$ & Pt-Con Thal. 0.78 & -3.49 & 0.001 & Pt-Con Hipp. & 0.12 & 0.54 & 0.589 \\
\hline 58 & -59 & 16 & cortex right & angular gyrus & $300 \mathrm{~mm} 2$ & Pt-Con Thal. 0.60 & -2.70 & 0.009 & Pt-Con Hipp. & 0.39 & 1.75 & 0.084 \\
\hline 68 & -9 & 21 & cortex right & postcentral gyrus & $488 \mathrm{~mm} 2$ & Pt-Con Thal. 0.63 & -2.84 & 0.006 & Pt-Con Hipp. & 0.54 & 2.43 & 0.018 \\
\hline 59 & -12 & 47 & corte & prece & & on Thal. 0.63 & -2.83 & 0.006 & Pt-Con Hipp. & 0.42 & 1.88 & 0.064 \\
\hline 60 & -4 & 34 & corte & & & Pt-Con Thal. 0.49 & -2.20 & 0.031 & Pt-Con Hipp. & 0.56 & 2.51 & 0.014 \\
\hline 56 & -7 & 13 & & & & Pt-Con Thal. 0.41 & -1.82 & 0.072 & Pt-Con Hipp. & 0.64 & 2.86 & 0.005 \\
\hline 55 & 4 & -14 & corte & medial t & $196 \mathrm{~m}$ & Pt-Con Thal. 0.61 & -2.75 & 0.007 & Pt-Con Hipp. & 0.57 & 2.55 & 0.013 \\
\hline 50 & -17 & 3 & cortex right & medial temporal lobe & $196 \mathrm{~mm} 2$ & Pt-Con Thal. 0.49 & -2.19 & 0.031 & Pt-Con Hipp. & 0.63 & 2.84 & 0.006 \\
\hline 61 & -7 & -2 & cortex right & medial temporal lobe & $256 \mathrm{~mm} 2$ & Pt-Con Thal. 0.55 & -2.48 & 0.015 & Pt-Con Hipp. & 0.62 & 2.79 & 0.007 \\
\hline 65 & -17 & 3 & cortex right & superior temporal gyrus & $244 \mathrm{~mm} 2$ & Pt-Con Thal. 0.51 & -2.27 & 0.026 & Pt-Con Hipp. & 0.77 & 3.48 & 0.001 \\
\hline-36 & -70 & & subcortex & left cerebellum & $200 \mathrm{~mm} 3$ & Pt-Con Thal. 0.54 & 2.43 & 0.017 & Pt-Con Hipp. & 0.77 & -3.48 & 0.001 \\
\hline
\end{tabular}


bioRxiv preprint doi: https://doi.org/10.1101/226951; this version posted November 29, 2017. The copyright holder for this preprint (which was not certified by peer review) is the author/funder, who has granted bioRxiv a license to display the preprint in perpetuity. It is made available under aCC-BY-ND 4.0 International license.

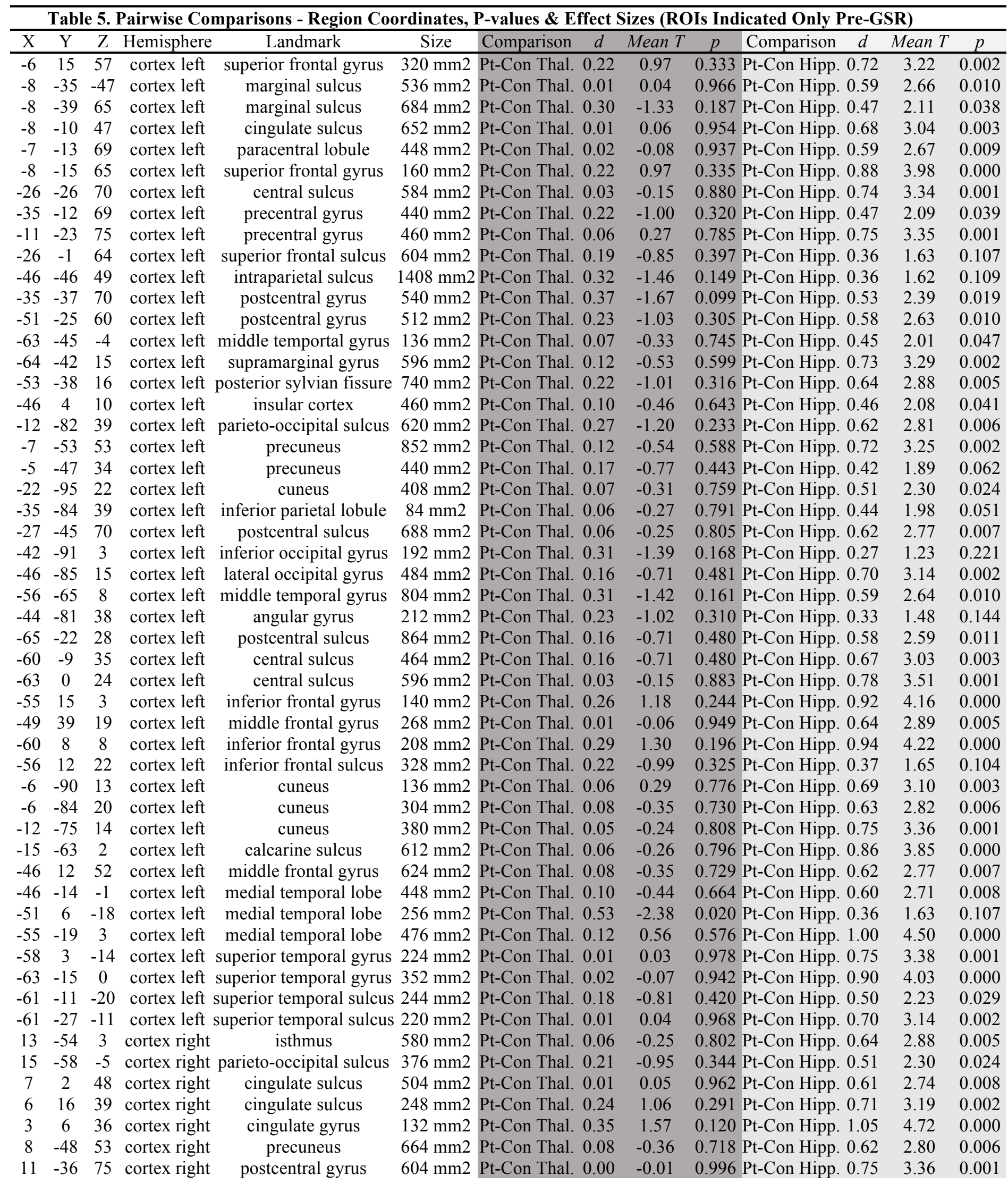


bioRxiv preprint doi: https://doi.org/10.1101/226951; this version posted November 29, 2017. The copyright holder for this preprint (which was not certified by peer review) is the author/funder, who has granted bioRxiv a license to display the preprint in perpetuity. It is made available under aCC-BY-ND 4.0 International license.

Main Text

$6 \quad-23 \quad 59$ cortex right

$\begin{array}{llll}6 & -8 & 61 & \text { cortex right }\end{array}$

$\begin{array}{llll}40 & -18 & 62 & \text { cortex right }\end{array}$

$\begin{array}{llll}44 & -3 & 61 & \text { cortex right }\end{array}$

$\begin{array}{llll}28 & -17 & 74 & \text { cortex right }\end{array}$

$\begin{array}{llll}12 & -14 & 71 & \text { cortex right }\end{array}$

$23 \quad 15 \quad 65$ cortex right superior frontal sulcus

$\begin{array}{llll}32 & -30 & 71 & \text { cortex right }\end{array}$

$\begin{array}{llll}42 & -34 & 0 & \text { cortex right }\end{array}$

$\begin{array}{llll}50 & -14 & 54 & \text { cortex right }\end{array}$

paracentral lobule

paracentral lobule

precentral gyrus

precentral gyrus

precentral gyrus

precentral sulcus

postcentral gyrus

central sulcus

postcentral gyrus

$\begin{array}{llll}64 & -38 & 0 & \text { cortex right middle temporal gyrus }\end{array}$

$\begin{array}{llll}62 & -46 & 24 & \text { cortex right superior temporal sulcus } 664 \mathrm{~mm} 2\end{array}$

$\begin{array}{llll}68 & -30 & 9 & \text { cortex right superior temporal gyrus } 376 \mathrm{~mm} 2 \\ \mathrm{Pt}-\mathrm{Con} \text { Thal } 0.16\end{array}$

$57-23 \quad 10$ cortex right medial temporal lobe

$\begin{array}{llll}56 & -32 & -1 & \text { cortex right medial temporal lobe }\end{array}$

$44-23 \quad 16$ cortex right insular cortex

$4948 \quad 5$ cortex right inferior frontal gyrus

$\begin{array}{llll}45 & -5 & -2 & \text { cortex right }\end{array}$

$\begin{array}{llll}44 & 10 & -8 & \text { cortex right }\end{array}$

insular cortex

insular cortex

orbital gyrus

$\begin{array}{cccc}8 & -67 & 28 & \text { cortex right parieto-occipital sulcus }\end{array}$

$\begin{array}{lllll}18 & -59 & 63 & \text { cortex right precuneus }\end{array}$

$\begin{array}{llll}21 & -76 & 47 & \text { cortex right parieto-occipital sulcus }\end{array}$

$\begin{array}{llll}34 & -51 & 43 & \text { cortex right intraparietal sulcus }\end{array}$

$29-70 \quad 53$ cortex right superior parietal lobule

$\begin{array}{llll}47 & -59 & 19 & \text { cortex right angular gyrus }\end{array}$

$\begin{array}{llll}62 & -34 & 47 & \text { cortex right supramarginal gyrus }\end{array}$

$\begin{array}{llll}65 & -14 & 18 & \text { cortex right }\end{array}$

$53-25 \quad 40$ cortex right

$\begin{array}{llll}65 & -1 & 25 & \text { cortex right }\end{array}$

postcentral gyrus

postcentral sulcus

central sulcus

$\begin{array}{llll}59 & -11 & 44 & \text { cortex right }\end{array}$

$\begin{array}{llll}52 & 34 & 22 & \text { cortex right }\end{array}$

postcentral gyrus

middle frontal gyrus

$\begin{array}{llll}60 & 10 & 7 & \text { cortex right precentral gyrus }\end{array}$

552120 cortex right inferior frontal sulcus

$\begin{array}{llll}20 & 31 & -19 & \text { cortex right }\end{array}$

$\begin{array}{llll}6 & -92 & 9 & \text { cortex right }\end{array}$

$\begin{array}{llll}11 & -78 & 4 & \text { cortex right }\end{array}$

$\begin{array}{llll}6 & -80 & 31 & \text { cortex right }\end{array}$

orbital gyrus

cuneus

calcarine sulcus

cuneus

$23-63 \quad 16$ cortex right parieto-occipital sulcus

$\begin{array}{llll}28 & 64 & 12 & \text { cortex right middle frontal gyrus }\end{array}$

$\begin{array}{llll}7 & 66 & 1 & \text { cortex right superior frontal gyrus }\end{array}$

156522 cortex right superior frontal gyrus $248 \mathrm{~mm} 2$ Pt-Con Thal. 0.11

$30 \quad 50 \quad 36$ cortex right superior frontal gyrus

54549 cortex right precentral gyrus

$46 \quad 8 \quad-17$ cortex right medial temporal lobe $224 \mathrm{~mm} 2$ Pt-Con Thal. 0.36

$\begin{array}{llll}54 & 8 & -16 & \text { cortex right superior temporal gyrus } 112 \mathrm{~mm} 2 \\ \text { Pt-Con Thal. } 0.35\end{array}$

$\begin{array}{llll}67 & -13 & 5 & \text { cortex right superior temporal gyrus } 268 \mathrm{~mm} 2 \\ \mathrm{Pt}-\mathrm{Con} \text { Thal. } 0.10\end{array}$

$59 \quad 0 \quad-20$ cortex right superior temporal sulcus $336 \mathrm{~mm} 2 \mathrm{Pt}-\mathrm{Con}$ Thal. 0.38
$424 \mathrm{~mm} 2$ Pt-Con Thal. 0.28

mm2 Pt-Con Thal. 0.07

$120 \mathrm{~mm} 2 \mathrm{Pt}-\mathrm{Con}$ Thal. 0.09

$408 \mathrm{~mm} 2$ Pt-Con Thal. 0.18

$356 \mathrm{~mm} 2$ Pt-Con Thal. 0.46

0.10

$432 \mathrm{~mm} 2 \mathrm{Pt}-\mathrm{Con}$ Thal. 0.02

Pt-Con Thal. 0.02

$556 \mathrm{~mm} 2 \mathrm{Pt}-$ Con Thal. 0.22

$316 \mathrm{~mm} 2 \mathrm{Pt}-\mathrm{Con}$ Thal. 0.09

$68 \mathrm{~mm} 2$ Pt-Con Thal. 0.13

276 mm2 Pt-Con Thal. 0.17

0.24

$24 \mathrm{~mm} 2$ Pt-Con Thal. 0.28

$80 \mathrm{~mm} 2$ Pt-Con Thal. 0.34

0.06

$684 \mathrm{~mm} 2 \mathrm{Pt}-\mathrm{Con}$ Thal 0.15

$508 \mathrm{~mm} 2$ Pt-Con Thal. 0.28

$20 \mathrm{~mm} 2$ Pt-Con Thal. 0.28

$572 \mathrm{~mm} 2$ Pt-Con Thal. 0.29

$104 \mathrm{~mm} 2$ Pt-Con Thal. 0.10

Pt-Con Thal. 0.09

$176 \mathrm{~mm} 2$ Pt-Con Thal. 0.04

$384 \mathrm{~mm} 2$ Pt-Con Thal. 0.03

$508 \mathrm{~mm} 2$ Pt-Con Thal 0.22

$460 \mathrm{~mm} 2$ Pt-Con Thal 0.25

$-1.5$

$-1.72$

\begin{tabular}{|c|c|c|c|}
\hline-1.27 & 0.209 Pt-Con Hip & & \\
\hline-0.31 & 0.756 Pt-Con Hipp. 0.50 & & \\
\hline & 1 Pt-Con $\mathrm{F}$ & & \\
\hline 0.42 & 75 Pt-Cor & & \\
\hline .79 & $0 \mathrm{Pt}-$ & & \\
\hline-0.44 & 63 Pt-Cor & & \\
\hline-2.08 & p. 0.13 & & \\
\hline-0.43 & 670 Pt_Co & & \\
\hline 1.63 & 107 Pt-Cor & 5 & \\
\hline & $\mathrm{t}-\mathrm{Cc}$ & & \\
\hline 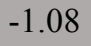 & $85 \mathrm{Pt}-\mathrm{Co}$ & & \\
\hline 10 & $\mathrm{t}-\mathrm{Co}$ & & \\
\hline-0.73 & $469 \mathrm{Pt}-\mathrm{Co}$ & & \\
\hline 0.09 & 30 & 5 & \\
\hline 0.09 & 0.72 & & \\
\hline 0.30 & 0.71 & 1 & \\
\hline 1 & 50 & & \\
\hline-0.39 & $6 \mathrm{Pt}-\mathrm{Cc}$ & 8 & \\
\hline 0.59 & 1 & & \\
\hline & 8 & & \\
\hline-0.78 & 0.52 & 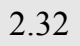 & \\
\hline-1. & 84 Pt-C & & \\
\hline-1.05 & 4 & 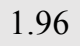 & \\
\hline-1.24 & $17 \mathrm{Pt}-\mathrm{C}$ & & \\
\hline-0.71 & $177 \mathrm{Pt}-\mathrm{Cc}$ & 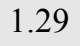 & \\
\hline-1.26 & $212 \mathrm{Pt}-\mathrm{Cc}$ & & \\
\hline & $29 \mathrm{Pt}-\mathrm{Cc}$ & & \\
\hline-0.26 & 4 & 7 & \\
\hline & 0 & & \\
\hline-0.68 & 98 & 3. & \\
\hline-1.28 & $05 \mathrm{Pt}_{-} \mathrm{C}$ & & \\
\hline-1.2 & $\mathrm{t}-\mathrm{C}$ & & \\
\hline 0.64 & 76 & 3 & \\
\hline-1.31 & $\mathrm{t}-\mathrm{C}$ & & \\
\hline-0.46 & & & \\
\hline-0.42 & 4 & & \\
\hline & 0 & & \\
\hline & & & \\
\hline 0.02 & 3 & 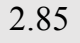 & \\
\hline 0.16 & 3 & 4 & \\
\hline-0.94 & $\mathrm{t}-\mathrm{C}$ & & \\
\hline & $5 \mathrm{Pt}-\mathrm{Cc}$ & & \\
\hline-0.51 & 0 & & \\
\hline & $34 \mathrm{Pt}-\mathrm{Cc}$ & 1.77 & \\
\hline & $0.261 \mathrm{Pt}-\mathrm{Co}$ & & \\
\hline 164 & $0.105 \mathrm{Pt}-\mathrm{Cor}$ & 2.33 & \\
\hline & 0.122 Pt-Cor & 1.77 & \\
\hline & 0.647 Pt-Cor & 3.18 & \\
\hline-1.72 & 090 Pt-Con Hipp. 0.37 & 1.67 & \\
\hline
\end{tabular}


bioRxiv preprint doi: https://doi.org/10.1101/226951; this version posted November 29, 2017. The copyright holder for this preprint (which was not certified by peer review) is the author/funder, who has granted bioRxiv a license to display the preprint in perpetuity. It is made available under aCC-BY-ND 4.0 International license.

Main Text

Running Title: Thalamic \& Hippocampal Dysconnectivity in 22q11DS

\section{REFERENCES}

Anticevic A, Lisman J (2017) How Can Global Alteration of Excitation/Inhibition Balance Lead to the Local Dysfunctions That Underlie Schizophrenia? Biol Psychiatry.

Anticevic A, Murray JD, Barch DM (2015a) Bridging Levels of Understanding in Schizophrenia Through Computational Modeling. Clinical Psychological Science 3:433-459.

Anticevic A, Cole MW, Repovs G, Murray JD, Brumbaugh MS, Winkler AM, Savic A, Krystal JH, Pearlson GD, Glahn DC (2014) Characterizing Thalamo-Cortical Disturbances in Schizophrenia and Bipolar Illness. Cereb Cortex 24:3116-3130.

Anticevic A et al. (2015b) Association of Thalamic Dysconnectivity and Conversion to Psychosis in Youth and Young Adults at Elevated Clinical Risk. JAMA Psychiatry [Epub].

Bassett AS, Chow EW (2008) Schizophrenia and 22q11.2 deletion syndrome. Curr Psychiatry Rep 10:148-157. Behrens TE, Johansen-Berg H, Woolrich MW, Smith SM, Wheeler-Kingshott CA, Boulby PA, Barker GJ, Sillery EL, Sheehan K, Ciccarelli O, Thompson AJ, Brady JM, Matthews PM (2003) Non-invasive mapping of connections between human thalamus and cortex using diffusion imaging. Nat Neurosci 6:750-757.

Bolkan SS, Stujenske JM, Parnaudeau S, Spellman TJ, Rauffenbart C, Abbas AI, Harris AZ, Gordon JA, Kellendonk C (2017) Thalamic projections sustain prefrontal activity during working memory maintenance. Nat Neurosci 20:987-996.

Brennand KJ, Simone A, Tran N, Gage FH (2012) Modeling psychiatric disorders at the cellular and network levels. Mol Psychiatry 17:1239-1253.

Brown EC, Clark DL, Hassel S, MacQueen G, Ramasubbu R (2017) Thalamocortical connectivity in major depressive disorder. J Affect Disord 217:125-131.

Buckner RL, Krienen FM, Castellanos A, Diaz JC, Yeo BT (2011) The organization of the human cerebellum estimated by intrinsic functional connectivity. J Neurophysiol 106:2322-2345.

Choi EY, Yeo BT, Buckner RL (2012) The organization of the human striatum estimated by intrinsic functional connectivity. J Neurophysiol 108:2242-2263.

Chun S, Westmoreland JJ, Bayazitov IT, Eddins D, Pani AK, Smeyne RJ, Yu J, Blundon JA, Zakharenko SS (2014) Specific disruption of thalamic inputs to the auditory cortex in schizophrenia models. Science 344:1178-1182.

Chun S, Du F, Westmoreland JJ, Han SB, Wang Y-D, Eddins D, Bayazitov IT, Devaraju P, Yu J, Mellado Lagarde MM, Anderson K, Zakharenko SS (2016) Thalamic miR-338-3p mediates auditory thalamocortical disruption and its late onset in models of 22q11.2 microdeletion. Nat Med advance online publication.

Cronenwett WJ, Csernansky J (2010) Thalamic pathology in schizophrenia. Current Topics in Behavioral Neurosciences 4:509-528.

Debbane M, Lazouret M, Lagioia A, Schneider M, Van De Ville D, Eliez S (2012) Resting-state networks in adolescents with 22q11.2 deletion syndrome: associations with prodromal symptoms and executive functions. Schizophr Res 139:33-39.

Earls LR, Zakharenko SS (2014) A synaptic function approach to investigating complex psychiatric diseases. The Neuroscientist : a review journal bringing neurobiology, neurology and psychiatry 20:257-271.

Eklund A, Nichols TE, Knutsson H (2016) Cluster failure: Why fMRI inferences for spatial extent have inflated false-positive rates. Proceedings of the National Academy of Sciences 113:7900-7905.

First MB, Spitzer RL, Gibbon M, Williams JBW (1996) Structured Clinical Interview for DSM-IV Axis I Disorders, Version 2.0 Edition. New York, NY: Biomedical Research Department, New York State Psychiatric Institute.

Geschwind DH, Flint J (2015) Genetics and genomics of psychiatric disease. Science 349:1489-1494. 
Glasser MF, Coalson TS, Bijsterbosch JD, Harrison SJ, Harms MP, Anticevic A, Van Essen DC, Smith SM (2017) Using Temporal ICA to Selectively Remove Global Noise While Preserving Global Signal in Functional MRI Data. bioRxiv.

Glasser MF, Coalson TS, Robinson EC, Hacker CD, Harwell J, Yacoub E, Ugurbil K, Andersson J, Beckmann CF, Jenkinson M, Smith SM, Van Essen DC (2016) A multi-modal parcellation of human cerebral cortex. Nature 536:171-178.

Glasser MF, Sotiropoulos SN, Wilson JA, Coalson TS, Fischl B, Andersson JL, Xu J, Jbabdi S, Webster M, Polimeni JR, Van Essen DC, Jenkinson M, Consortium W-MH (2013) The Minimal Preprocessing Pipelines for the Human Connectome Project. Neuroimage 80.

Gonzalez-Burgos G, Lewis DA (2012) NMDA Receptor Hypofunction, Parvalbumin-Positive Neurons and Cortical Gamma Oscillations in Schizophrenia. Schizophr Bull 38:950-957.

Green T, Gothelf D, Glaser B, Debbane M, Frisch A, Kotler M, Weizman A, Eliez S (2017) Psychiatric Disorders and Intellectual Functioning Throughout Development in Velocardiofacial (22q11.2 Deletion) Syndrome. J Am Acad Child Adolesc Psychiatry 48:1060-1068.

Guo ZV, Inagaki HK, Daie K, Druckmann S, Gerfen CR, Svoboda K (2017) Maintenance of persistent activity in a frontal thalamocortical loop. Nature advance online publication.

Haber S, McFarland NR (2001) The place of the thalamus in frontal cortical-basal ganglia circuits. Neuroscientist 7:315-324.

Hallock HL, Wang A, Griffin AL (2016) Ventral Midline Thalamus Is Critical for Hippocampal-Prefrontal Synchrony and Spatial Working Memory. J Neurosci 36:8372-8389.

Herkenham M (1978) The connections of the nucleus reuniens thalami: evidence for a direct thalamohippocampal pathway in the rat. J Comp Neurol 177:589-610.

Hiroi N, Takahashi T, Hishimoto A, Izumi T, Boku S, Hiramoto T (2013) Copy number variation at 22q11.2: from rare variants to common mechanisms of developmental neuropsychiatric disorders. Mol Psychiatry 18:1153-1165.

Hwang K, Bertolero M, Liu W, Esposito M (2017) The human thalamus is an integrative hub for functional brain networks. The Journal of Neuroscience.

Insel TR (2010) Rethinking schizophrenia. Nature 468:187-193.

Jalbrzikowski M, Jonas R, Senturk D, Patel A, Chow C, Green MF, Bearden CE (2013) Structural abnormalities in cortical volume, thickness, and surface area in 22q11.2 microdeletion syndrome: Relationship with psychotic symptoms(). NeuroImage : Clinical 3:405-415.

Jalbrzikowski M, Villalon-Reina JE, Karlsgodt KH, Senturk D, Chow C, Thompson PM, Bearden CE (2014) Altered white matter microstructure is associated with social cognition and psychotic symptoms in 22q11.2 microdeletion syndrome. Frontiers in Behavioral Neuroscience 8:393.

Jalbrzikowski M, Carter C, Senturk D, Chow C, Hopkins JM, Green MF, Galván A, Cannon TD, Bearden CE (2012) Social Cognition in 22q11.2 Microdeletion Syndrome: Relevance to Psychosis. Schizophr Res 142:99-107.

Javitt DC (2007) Glutamate and schizophrenia: phencyclidine, N-methyl-D-aspartate receptors, and dopamineglutamate interactions. Int Rev Neurobiol 78:69-108.

Jenkinson M, Bannister P, Brady M, Smith S (2002) Improved optimization for the robust and accurate linear registration and motion correction of brain images. Neuroimage 17:825-841.

Kates WR, Burnette CP, Jabs EW, Rutberg J, Murphy AM, Grados M, Geraghty M, Kaufmann WE, Pearlson GD (2001) Regional cortical white matter reductions in velocardiofacial syndrome: a volumetric MRI analysis. Biol Psychiatry 49:677-684.

Lewis DA, Curley AA, Glausier JR, Volk DW (2012) Cortical parvalbumin interneurons and cognitive dysfunction in schizophrenia. Trends Neurosci 35:57-67. 
Lisman J (2012) Excitation, inhibition, local oscillations, or large-scale loops: what causes the symptoms of schizophrenia? Curr Opin Neurobiol 22:537-544.

Loh M, Rolls ET, Deco G (2007) A dynamical systems hypothesis of schizophrenia. PLoS Comput Biol 3:e228. McDonald-McGinn DM, Sullivan KE, Marino B, Philip N, Swillen A, Vorstman JAS, Zackai EH, Emanuel BS, Vermeesch JR, Morrow BE, Scambler PJ, Bassett AS (2015) 22q11.2 deletion syndrome. Nature Reviews Disease Primers 1:15071.

Meechan D, Tucker E, Maynard T, LaMantia AS (2012) Cxcr4 regulation of interneuron migration is disrupted in 22q11.2 deletion syndrome. Proceedings of the National Academy of Sciences 109:18601-18606.

Meechan D, Maynard T, Tucker E, Fernandez A, Karpinski B, Rothblat L, LaMantia A (2015) Modeling a model: Mouse genetics, 22q11.2 Deletion Syndrome, and disorders of cortical circuit development. Prog Neurobiol 130:1-28.

Meyer-Lindenberg A (2010) From maps to mechanisms through neuroimaging of schizophrenia. Nature 468:194-202.

Mukai J, Tamura M, Fenelon K, Rosen AM, Spellman TJ, Kang R, MacDermott AB, Karayiorgou M, Gordon JA, Gogos JA (2015) Molecular substrates of altered axonal growth and brain connectivity in a mouse model of schizophrenia. Neuron 86:680-695.

Oke AF, Adams RN, Winblad B, von Knorring L (1988) Elevated dopamine/norepinephrine ratios in thalami of schizophrenic brains. Biol Psychiatry 24:79-82.

Padula MC, Schaer M, Scariati E, Schneider M, Van De Ville D, Debbané M, Eliez S (2015) Structural and functional connectivity in the default mode network in 22q11.2 deletion syndrome. J Neurodev Disord $7: 23$.

Paronett EM, Meechan DW, Karpinski BA, LaMantia AS, Maynard TM (2015) Ranbp1, Deleted in DiGeorge/22q11.2 Deletion Syndrome, is a Microcephaly Gene That Selectively Disrupts Layer 2/3 Cortical Projection Neuron Generation. Cereb Cortex 25:3977-3993.

Power JD, Plitt M, Laumann TO, Martin A (2017) Sources and implications of whole-brain fMRI signals in humans. Neuroimage 146:609-625.

Power JD, Barnes KA, Snyder AZ, Schlaggar BL, Petersen SE (2012) Spurious but systematic correlations in functional connectivity MRI networks arise from subject motion. Neuroimage 59:2142-2154.

Reuter M, Schmansky NJ, Rosas HD, Fischl B (2012) Within-subject template estimation for unbiased longitudinal image analysis. Neuroimage 61:1402-1418.

Samudra N, Ivleva EI, Hubbard NA, Rypma B, Sweeney JA, Clementz BA, Keshavan MS, Pearlson GD, Tamminga CA (2015) Alterations in hippocampal connectivity across the psychosis dimension. Psychiatry Res 233:148-157.

Schmitt JE, Vandekar S, Yi J, Calkins ME, Ruparel K, Roalf DR, Whinna D, Souders MC, Satterthwaite TD, Prabhakaran K, McDonald-McGinn DM, Zackai EH, Gur RC, Emanuel BS, Gur RE (2015) Aberrant Cortical Morphometry in the 22q11.2 Deletion Syndrome. Biol Psychiatry 78:135-143.

Schneider M et al. (2014) Psychiatric Disorders From Childhood to Adulthood in 22q11.2 Deletion Syndrome: Results From the International Consortium on Brain and Behavior in 22q11.2 Deletion Syndrome. Am J Psychiatry 171:627-639.

Schreiner M, Forsyth JK, Karlsgodt KH, Anderson AE, Hirsh N, Kushan L, Uddin LQ, Mattiacio L, Coman IL, Kates WR, Bearden CE (2017) Intrinsic Connectivity Network-Based Classification and Detection of Psychotic Symptoms in Youth With 22q11.2 Deletions. Cereb Cortex:1-13.

Sigurdsson T, Stark KL, Karayiorgou M, Gogos JA, Gordon JA (2010) Impaired hippocampal-prefrontal synchrony in a genetic mouse model of schizophrenia. Nature 464:763-767.

Steiger J (1980) Tests for Comparing Elements of a Correlation Matrix. 
Stein T, Moritz C, Quigley M, Cordes D, Haughton V, Meyerand E (2000) Functional Connectivity in the Thalamus and Hippocampus Studied with Functional MR Imaging. American Journal of Neuroradiology 21:1397-1401.

Tamminga CA, Stan AD, Wagner AD (2010) The hippocampal formation in schizophrenia. Am J Psychiatry 167:1178-1193.

Thorndike RL (1953) Who belongs in the family? Psychometrika 18:267-276.

Toritsuka M, Kimoto S, Muraki K, Landek-Salgado MA, Yoshida A, Yamamoto N, Horiuchi Y, Hiyama H, Tajinda K, Keni N, Illingworth E, Iwamoto T, Kishimoto T, Sawa A, Tanigaki K (2013) Deficits in microRNA-mediated Cxcr4/Cxcl12 signaling in neurodevelopmental deficits in a 22q11 deletion syndrome mouse model. Proc Natl Acad Sci U S A 110:17552-17557.

Weinberger DR (1987) Implications of normal brain development for the pathogenesis of schizophrenia. Arch Gen Psychiatry 44:660-669.

Welsh RC, Chen AC, Taylor SF (2010) Low-frequency BOLD fluctuations demonstrate altered thalamocortical connectivity in schizophrenia. Schizophr Bull 36:713-722.

Winkler AM, Ridgway GR, Webster MA, Smith SM, Nichols TE (2014) Permutation inference for the general linear model. Neuroimage 92:381-397.

Woodward ND, Karbasforoushan H, Heckers S (2012) Thalamocortical dysconnectivity in schizophrenia. Am J Psychiatry 169:1092-1099.

Yeo BT, Krienen FM, Sepulcre J, Sabuncu MR, Lashkari D, Hollinshead M, Roffman JL, Smoller JW, Zollei L, Polimeni JR, Fischl B, Liu H, Buckner RL (2011) The organization of the human cerebral cortex estimated by intrinsic functional connectivity. J Neurophysiol 106:1125-1165.

Zhang D, Snyder AZ, Shimony JS, Fox MD, Raichle ME (2010) Noninvasive functional and structural connectivity mapping of the human thalamocortical system. Cereb Cortex 20:1187-1194. 